\title{
MODELLING OPTIMAL INSTRUMENTAL VARIABLES FOR DYNAMIC PANEL DATA MODELS
}

\author{
Manuel Arellano \\ CEMFI Working Paper No. 0310 \\ July 2003 \\ CEMFI \\ Casado del Alisal 5, 28014 Madrid \\ Tel. (34) 914290 551. Fax (34) 914291056 \\ Internet: www.cemfi.es
}

An earlier version of this paper was presented as an Econometrics Invited Lecture at the European Meeting of the Econometric Society in Venice, August 2002. I wish to thank Stephen Bond, Francesco Caselli, and Jonathan Temple for kindly making available their datasets; Pedro Albarran for outstanding research assistance; Javier Alvarez, Olympia Bover, Jan Kiviet, Charles Manski, and Enrique Sentana for helpful comments, and David Hendry for posing the question that originated this work. 
CEMFI Working Paper No. 0310

July 2003

\title{
MODELLING OPTIMAL INSTRUMENTAL VARIABLES FOR DYNAMIC PANEL DATA MODELS
}

\begin{abstract}
Two-step instrumental variable estimators for dynamic panel data models are considered that are asymptotically efficient under some auxiliary assumptions, but remain consistent when the assumptions are violated. Asymptotic efficiency is defined in relation to the information bound for the conditional mean specification of the model. Unlike in standard panel GMM, optimal instruments are parameterized using a fixed number of coefficients for any value of $T$. Thus, the properties of the resulting estimators are not fundamentally affected by the relative dimensions of $T$ and $N$.
\end{abstract}

JEL Codes: C23

Keywords: Dynamic panel data, optimal instruments, GMM, double asymptotics, cross-country growth

Manuel Arellano

CEMFI

arellano@cemfi.es 


\section{Introduction}

In this paper we discuss a new method for estimating panel data models with general predetermined or endogenous explanatory variables. In doing so we propose a framework for modelling optimal instruments in panel data analysis.

A popular method in dynamic panel data estimation is GMM, which is consistent in short panels, robust, has general applicability, and provides a well defined notion of optimality (Holtz-Eakin, Newey and Rosen, 1988; Arellano and Bond, 1991). However, in practice the application of GMM often entails too many instruments for acceptable sampling properties in either finite or large samples when the time series dimension is not fixed (Alvarez and Arellano, 2003).

For autoregressive models there are also available likelihood-based methods which exhibit better finite sample properties than GMM but can be seriously biased if certain auxiliary assumptions are violated. Moreover, these methods cannot be readily extended to cover models with endogenous or general predetermined variables. There is therefore an acute robustness-efficiency trade-off in the choice among existing techniques. In addition, some methods are designed for short panels of large cross-sections, while others target long panels of small cross-sections, but there is a vacuum in between. The literature does not seem to have much to offer to researchers interested in "small $N$, small $T$ " panels or other panels that are not easily classifiable.

GMM estimators can be regarded as providing implicit models for the optimal instruments. The problem is that in the panel context these models are often overparameterized, leading to poor properties in finite samples and double asymptotics. This paper provides a framework for parsimonious modelling of optimal instruments in panel data, which is, first, coherent with the fixed $T$, large $N$ perspective; second, has good theoretical properties in a double asymptotic 
setup, and thirdly provides estimators with the same robustness features as popular GMM methods. More research is needed on panel data methodology from a time series perspective, and this paper is intended as a contribution towards a marriage of the cross-sectional (fixed $T$ ) and time-series (long $T$ ) perspectives.

The paper is organized as follows. Section 2 presents the model, links GMM with the parametric optimal-instrument perspective, and introduces projectionrestricted simple IV estimation (SIV). Section 3 discusses the asymptotic biases of one-step GMM when both $T$ and $N$ tend to infinity. It is shown that the order of magnitude of the bias depends on whether the explanatory variables are predetermined or endogenous, and that in the latter situation GMM is inconsistent. In Section 4 we present an auxiliary random effects VAR model for the vector of instruments, and obtain sequential linear projections of the effects. Section 5 describes the form of optimal instruments and provides several examples. Section 6 considers pseudo maximum likelihood estimation (PML) of the auxiliary VAR model. Section 7 discusses the properties of feasible projection-restricted IV estimators with and without completely endogenous variables, and the calculation of asymptotic variance matrix estimates. Section 8 contains empirical illustrations and Monte Carlo simulations. Subsection 8.1 reports estimates of autoregressive employment and wage equations from firm panel data; Subsection 8.2 presents the results of a simulation exercise calibrated to the previous firm panel, and Subsection 8.3 reports estimates of country growth convergence rates using a panel of 92 countries observed at five-year intervals. Finally, Section 9 ends with some concluding remarks and plans for future work. All proofs and technical details are contained in Appendices. 


\section{Model and Optimal Instruments: Overview}

\subsection{A Sequential Conditional Mean Model}

Let us consider a fixed effects panel data model of the form

$$
y_{i t}=x_{i t}^{\prime} \beta+\eta_{i}+\varepsilon_{i t}(t=1, \ldots, T ; i=1, \ldots, N),
$$

together with the conditional mean assumption

$$
E\left(\varepsilon_{i t} \mid z_{i}^{t}\right) \equiv E_{t}\left(\varepsilon_{i t}\right)=0
$$

where $z_{i}^{t}=\left(z_{i 1}^{\prime}, \ldots, z_{i t}^{\prime}\right)^{\prime}$ and $\left(y_{i}^{T}, x_{i}^{T}, z_{i}^{T}, \eta_{i}\right)$ are $i i d$ random variables; $\eta_{i}$ represents an unobservable individual effect and $z_{i t}$ is a vector of instrumental variables.

The following remarks about the nature of the explanatory variables and the instruments are relevant. First, if an explanatory variable $x_{k i t}$ is predetermined for $\varepsilon_{i(t+j)}$, then $x_{k i(t-j)}$ is a component of $z_{i t}$. Second, an $x_{k i t}$ may also be completely endogenous in the sense of not being predetermined for any lead of $\varepsilon$. Finally, $z_{i t}$ may contain external instruments that are not part of $x_{i t}$ or its lags.

An example of this type of model is an equation from a VAR with individual effects. Other examples are partial adjustment equations with predetermined regressors, or a structural relationship between endogenous variables. As illustrations of the latter we consider below cross-country growth and household consumption Euler equations.

\subsection{Information Bound and Optimal Instruments}

Since the distribution of $\eta_{i} \mid z_{i}^{T}$ is unrestricted, all information about $\beta$ is in the conditional moments for the errors in differences or forward orthogonal deviations

$$
E_{t}\left(y_{i t}^{*}-x_{i t}^{* \prime} \beta\right)=E_{t}\left(\varepsilon_{i t}^{*}\right)=0 \quad(t=1, \ldots, T-1)
$$


where starred variables denote orthogonal deviations:

$$
\varepsilon_{i t}^{*}=\left(\frac{T-t}{T-t+1}\right)^{1 / 2}\left[\varepsilon_{i t}-\frac{1}{(T-t)}\left(\varepsilon_{i(t+1)}+\ldots+\varepsilon_{i T}\right)\right] .
$$

The advantage of orthogonal deviations is that if $\varepsilon_{i t}$ is homoskedastic and serially uncorrelated so is $\varepsilon_{i t}^{*}$ (Arellano and Bover, 1995).

Two-wave Panel If $T=2$ there is just one equation in deviations (which coincides with first-differences):

$$
E\left(y_{i 1}^{*}-x_{i 1}^{* \prime} \beta \mid z_{i}^{1}\right)=E_{1}\left(\varepsilon_{i 1}^{*}\right)=0
$$

and the optimal instrument is $h_{i 1}=E_{1}\left(x_{i 1}^{*}\right) / E_{1}\left(\varepsilon_{i 1}^{* 2}\right)$, in the sense that the unfeasible IV estimator

$$
\widetilde{\beta}_{1}=\left(\sum_{i=1}^{N} h_{i 1} x_{i 1}^{* \prime}\right)^{-1} \sum_{i=1}^{N} h_{i 1} y_{i 1}^{*}
$$

attains the variance bound for this problem.

A parametric approach to feasible estimation is to specify functional forms for $E_{1}\left(x_{i 1}^{*}\right)$ and $E_{1}\left(\varepsilon_{i 1}^{* 2}\right)$ and substitute suitable estimates in the IV formula (2.6). 2SLS is an example of this approach that uses the sample linear projection of $x_{i 1}^{*}$ on $z_{i}^{1}$ as an estimate of the optimal instrument. That is, 2SLS attains the variance bound when $E_{1}\left(x_{i 1}^{*}\right)$ is linear and $E_{1}\left(\varepsilon_{i 1}^{* 2}\right)$ is constant. ${ }^{1}$

Thus, in a parametric approach to feasible IV estimation there are two levels of assumptions: the substantive conditional moment restrictions used in estimation, and the auxiliary assumptions used in estimating the optimal instruments. The former are related to consistency and the latter to asymptotic efficiency. This distinction between substantive and auxiliary assumptions is central to the perspective adopted in this paper.

\footnotetext{
${ }^{1}$ Incorporating these assumptions in estimation may reduce the variance bound for $\beta$. A trade-off between robustness and efficiency arises, since estimates of $\beta$ that exploit the extra restrictions may be inconsistent if they are false.
} 
Multi-wave Panel If $T>2$ the form of the optimal instrument is complicated by the fact that neither conditional heteroskedasticity or autocorrelation in $\varepsilon_{i t}^{*}$ are ruled out. The unfeasible optimal IV estimator in the general case solves

$$
\sum_{i=1}^{N} \sum_{t=1}^{T-1} h_{i t}\left(\widetilde{y}_{i t}-\widetilde{x}_{i t}^{\prime} \widetilde{\beta}\right)=0
$$

where $h_{i t}=E_{t}\left(\widetilde{x}_{i t}\right) / E_{t}\left(\widetilde{\varepsilon}_{i t}^{2}\right)$ and

$$
\begin{gathered}
\widetilde{\varepsilon}_{i(T-1)}=\varepsilon_{i(T-1)}^{*} \\
\widetilde{\varepsilon}_{i t}=\varepsilon_{i t}^{*}-\tau_{t 1} \widetilde{\varepsilon}_{i(t+1)}-\ldots-\tau_{t(T-t-1)} \widetilde{\varepsilon}_{i(T-1)}(t=T-2, \ldots, 1)
\end{gathered}
$$

with $\tau_{t j}=E_{t+j}\left(\varepsilon_{i t}^{*} \widetilde{\varepsilon}_{i(t+j)}\right) / E_{t+j}\left(\widetilde{\varepsilon}_{i(t+j)}^{2}\right)$.

The $\widetilde{\varepsilon}_{i t}$ are forward filtered errors such that $E_{t}\left(\widetilde{\varepsilon}_{i t}\right)=0$ and $E_{t+j}\left(\widetilde{\varepsilon}_{i t} \widetilde{\varepsilon}_{i(t+j)}\right)=0$ so that the bound for $T-1$ periods is the sum of the bounds for each period (Chamberlain, 1992):

$$
J_{T-1}=\sum_{t=1}^{T-1} E\left(\frac{E_{t}\left(\widetilde{x}_{i t}\right) E_{t}\left(\widetilde{x}_{i t}\right)^{\prime}}{E_{t}\left(\widetilde{\varepsilon}_{i t}^{2}\right)}\right) .
$$

If the $\varepsilon_{i t}^{*}$ 's are conditionally homoskedastic and serially uncorrelated $\widetilde{\varepsilon}_{i t}=\varepsilon_{i t}^{*}$. The optimal instrument is $h_{i t} \equiv h_{t}\left(z_{i}^{t}\right)=E_{t}\left(x_{i t}^{*}\right)$, and the unfeasible IVE

$$
\widetilde{\beta}=\left(\sum_{i=1}^{N} \sum_{t=1}^{T-1} h_{i t} x_{i t}^{* \prime}\right)^{-1}\left(\sum_{i=1}^{N} \sum_{t=1}^{T-1} h_{i t} y_{i t}^{*}\right)
$$

attains the fixed- $T$, large- $N$ variance bound.

Generalized Method of Moments A One-step GMM estimator (GMM1) is an example of the parametric approach to feasible IVE that uses the crosssectional sample linear projections of $x_{i t}^{*}$ on $z_{i}^{t}$ as an estimate of the optimal instruments. GMM1 is based on the specification

$$
h_{t}\left(z_{i}^{t}\right)=z_{i 1}^{\prime} \pi_{t 1}+\ldots+z_{i t}^{\prime} \pi_{t t} \equiv z_{i}^{t \prime} \pi_{t}
$$


together with assumptions of homoskedasticity and lack of serial correlation.

In a time series context (large $T$, fixed $N$ ), these projections cannot be consistently estimated without further restrictions. But as $N \rightarrow \infty$ for fixed $T$, the IVE that uses the sample projection $z_{i}^{t \prime} \widehat{\pi}_{t}$ has the same asymptotic distribution as the unfeasible IVE based on the population projection $z_{i}^{t \prime} \pi_{t}$.

However, when both $T$ and $N$ tend to infinity at the same rate, the feasible and unfeasible estimators differ as shown for autoregressive models in Alvarez and Arellano (2003), who found that GMM1 had an asymptotic bias of order $1 / N$. In the next Section we obtain the asymptotic bias of GMM1 for model (2.1)-(2.2) when both $T$ and $N$ tend to infinity. We show that the order of magnitude of the bias depends on whether the explanatory variables are endogenous or predetermined. In the predetermined case the bias is of order $1 / N$ - as in Alvarez and Arellano (2003) - but in the endogenous case the bias is of order $T / N$, and hence a potentially more serious problem. These results provide theoretical support for the approach developed in this paper, because the proposed estimators are immune to asymptotic bias in a double asymptotics.

Under heteroskedasticity or autocorrelation, it is possible to obtain a GMM estimator based on the moments $E\left(z_{i}^{t} v_{i t}^{*}\right)=0$ that is more efficient than $\widehat{\beta}_{G M M 1}$ by using as weight matrix the inverse of a robust estimate of the variance matrix of the orthogonality conditions. These are the standard two-step robust GMM2 estimators (Arellano and Bond, 1991). A GMM2 estimator, however, will not attain the efficiency bound in general, although it may attain it under more general conditions than GMM1. Specifically, GMM2 will attain the bound under serial correlation and time series heteroskedasticity of the form $E\left(v_{i t}^{2} \mid z_{i}^{t}\right)=E\left(v_{i t}^{2}\right)=\sigma_{t}^{2}$ and $E\left(v_{i t} v_{i(t+j)} \mid z_{i}^{t+j}\right)=E\left(v_{i t} v_{i(t+j)}\right)=\sigma_{t(t+j)}$ for $j>0 .^{2}$

\footnotetext{
${ }^{2}$ A more restrictive two-step GMM estimator based on a weight matrix that only depends on the data second-order moments (Arellano and Bond, 1991, footnote 2), will attain the bound under the same conditions as the standard GMM2.
} 
Semiparametric Asymptotically Efficient Estimation for Fixed $T$ It is also possible to devise estimators that attain the fixed- $T$ efficiency bound under more general assumptions than GMM1 or GMM2. This could be achieved by considering a semiparametric estimator that replaces the unknown functions in (2.11) or (2.7) by nonparametric estimates. The former would be asymptotically efficient when the conditional expectations $E\left(x_{i t}^{*} \mid z_{i}^{t}\right)$ are nonlinear but the errors are classical. The latter might be efficient in the general case. Estimators of this type have been considered by Hahn (1997). Nevertheless, since these estimators use even more flexible estimates of $E\left(x_{i t}^{*} \mid z_{i}^{t}\right)$ than GMM1, they would not be expected to perform better than GMM1 unless $T / N$ is sufficiently close to zero.

\subsection{The Crude Time-Series Parametric Approach}

The conventional time-series parametric approach specifies

$$
h_{t}\left(z_{i}^{t}\right)=\sum_{j=0}^{q} z_{i(t-j)}^{\prime} \gamma_{j}
$$

for some $q .{ }^{3}$ Equation (2.13) has constant coefficients $\gamma_{j}$ that can be consistently estimated time series-wise. It is based on assumptions about the stability and degree of dependence in the $z_{i t}$ process.

Panel data examples of the time-series approach are the stacked IV estimators for autoregressive models due to Anderson and Hsiao (1982). From the point of view of fixed- $T$ optimal IV estimation, however, the crude time-series approach has two undesirable features. First, (2.13) cannot be calculated for periods without sufficient observations of the initial lags, hence requiring trimming of the time series and loss of some cross-sections.

A second less obvious problem is that if the $z_{i t}$ process contains heterogeneous intercepts - as may be expected given model $(2.1) — h_{t}\left(z_{i}^{t}\right)$ will depend on all lags,

\footnotetext{
${ }^{3}$ In this case it is better to think of $h_{t}\left(z_{i}^{t}\right)$ as $E_{t}\left(x_{i t}-x_{i(t+1)}\right)$ because $x_{i t}^{*}$ involves a different number of terms for each $t$.
} 
even if at the individual level $z$ is characterized by stable low-order dependence of the kind that supports assumption (2.13) in a time series context. The reason is that all lags are predictors of the effects (as described in Section 4). Since the predictions are updated each period, the coefficients of the predictor change over time. This is precisely the motivation behind (2.12).

\subsection{Projection-Restricted IV Estimation}

The problem with $z_{i}^{t \prime} \pi_{t}$ is that it often contains too many coefficients for good finite sample inference. If we have an individual-effects, stable process for $z$ characterized by a parameter vector $\gamma$, the $\pi_{t}$ are functions of $\gamma$. Therefore, instead of an unrestricted linear projection we may consider a restricted one:

$$
h_{t}\left(z_{i}^{t}\right)=z_{i}^{t \prime} \pi_{t}(\gamma)
$$

These restrictions are similar to the low order, stable dependence assumptions implicit in the time-series approach. The difference is that we are using them in a fashion consistent with the fixed- $T$ panel perspective.

For a long- $T$ panel the outcome is essentially the same as in the crude timeseries approach. For a very short- $T$ panel the outcome is essentially the same as in the standard GMM approach. But for other panels the number of first-stage coefficients is kept constant, while using all waves and the predictive ability due to unobserved heterogeneity.

The suggested strategy is to specify a VAR for $z_{i t}$ with individual effects and unrestricted initial conditions. The status of this assumption is similar to that of (2.13) in the time-series approach. Then use as instruments the estimated restricted projections $z_{i}^{t \prime} \pi_{t}(\gamma)$, whose evaluation involves, as we shall see, a straightforward recursive Kalman-filter calculation.

Unrestricted $\pi_{t}$ in GMM are estimated by cross-sectional OLS. Restricted 
$\pi_{t}(\gamma)$ will be estimated using a modified WG estimator, which is a multivariate generalization of the random effects PML in Alvarez and Arellano (2003).

We then obtain

$$
\widehat{\beta}=\left(\sum_{i} \sum_{t} \widehat{h}_{i t} x_{i t}^{* \prime}\right)^{-1} \sum_{i} \sum_{t} \widehat{h}_{i t} y_{i t}^{*} .
$$

GMM sets $\widehat{h}_{i t}=z_{i}^{t \prime} \widehat{\pi}_{t}$ where $\widehat{\pi}_{t}$ is an OLS estimate, and the projection-restricted IVE sets $\widehat{h}_{i t}=z_{i}^{t \prime} \pi_{t}(\widehat{\gamma})$ where $\widehat{\gamma}$ is a PML estimate.

For fixed $T$ and large $N$ both estimators have similar robustness properties in the sense of being consistent under the same assumptions, but when $T$ is not fixed the latter is immune to asymptotic biases because the number of first-stage coefficients does not increase with $T$. If the auxiliary assumptions $\pi_{t}=\pi_{t}(\gamma)$ are violated, projection-restricted IV remains a consistent estimate of $\beta$.

The goal is, therefore, to use the time-series parametric approach in such a way that the resulting estimator can still achieve the fixed $T$ efficiency bound under certain assumptions, in the same way as standard GMM only achieves the fixed $T$ bound under certain auxiliary assumptions.

\section{Double-Asymptotic Biases}

In this Section we obtain the asymptotic bias of the one-step GMM estimator when both $T$ and $N$ tend to infinity. We show that the order of magnitude of the bias depends on whether the explanatory variables are endogenous or predetermined.

It is well known that if the number of first-stage coefficients is large relative to the sample size, standard asymptotic approximations may be a poor guide to the finite sample properties of IV estimates, specially when the instruments are weak. In the panel context, double-asymptotic results provide formal approximations to the impact of nonnegligible $T$ (and hence of many instruments) relative to $N$ 
on the properties of GMM estimates. They also provide a theoretical motivation for the strategy for reducing the number of first-stage coefficients pursued in this paper.

The form of the GMM estimator is

$$
\widehat{\beta}=\left(\sum_{t=1}^{T-1} X_{t}^{* \prime} M_{t} X_{t}^{*}\right)^{-1} \sum_{t=1}^{T-1} X_{t}^{* \prime} M_{t} y_{t}^{*},
$$

where $M_{t}=Z_{t}\left(Z_{t}^{\prime} Z_{t}\right)^{-1} Z_{t}^{\prime}$ is $N \times N, X_{t}^{*}=\left(x_{1 t}^{*}, \ldots, x_{N t}^{*}\right)^{\prime}$ is $N \times k, Z_{t}=\left(z_{1}^{t}, \ldots, z_{N}^{t}\right)^{\prime}$ is $N \times m t$, and $y_{t}^{*}=\left(y_{1 t}^{*}, \ldots, y_{N t}^{*}\right)^{\prime}$ is $N \times 1$, or in a more compact notation

$$
\widehat{\beta}=\left(X^{* \prime} M X^{*}\right)^{-1} X^{* \prime} M y^{*} .
$$

\subsection{Assumptions}

Let us define the vector of variables $w_{i t}=\left(\varepsilon_{i t}, x_{i t}^{\prime}, z_{i t}^{\prime}\right)^{\prime}$. Depending on the model, $x$ and $z$ may contain elements in common. Also, some of the $x$ s may be lags of $y$, and some of the $z$ s may be lags of $y$ and/or $x$. We make the following assumption: Assumption 1: $w_{i t}$ can be represented as a vector $\mathrm{MA}(\infty)$ of the form

$$
w_{i t}=\mu_{i}+\zeta_{i t}+\Psi_{1} \zeta_{i(t-1)}+\Psi_{2} \zeta_{i(t-2)}+\ldots
$$

where $\left\{\Psi_{j}\right\}_{j=0}^{\infty}$ is absolutely summable, and $\left\{\zeta_{i t}\right\}_{t=-\infty}^{\infty}$ is an i.i.d. sequence independent of the individual-specific mean vector $\mu_{i}$ and with finite fourth-order moments. Moreover, the first element of $\mu_{i}$ is zero, and

$$
\sum_{s=-\infty}^{\infty}|s| \gamma_{s}=B_{\infty}<\infty
$$

where $\gamma_{s}=E\left(x_{i t} \varepsilon_{i(t-s)}\right)$.

GMM estimators are typically motivated under less restrictive conditions than Assumption 1, which is only made for simplicity, since our purpose is to exhibit 
some consequences of double-asymptotics in a leading situation, rather than providing an alternative basis for inference with GMM estimates. The results that follow are expected to hold under more general conditions.

Predeterminedness and Endogeneity If $x_{i t}$ is predetermined for $\varepsilon_{i t}$, then $x_{i t} \vdash\left(\varepsilon_{i t}, \varepsilon_{i(t+1)}, \varepsilon_{i(t+2)}, \ldots\right)$ but $x_{i t} \nvdash\left(\varepsilon_{i(t-1)}, \varepsilon_{i(t-2)}, \ldots\right)$, so that $\gamma_{0}=\gamma_{-1}=\ldots=0$ but $\gamma_{s} \neq 0$ for $s>0$. If on the other hand $x_{i t}$ is completely endogenous

$$
x_{i t} \nvdash\left(\ldots, \varepsilon_{i(t-2)}, \varepsilon_{i(t-1)}, \varepsilon_{i t}, \varepsilon_{i(t+1)}, \varepsilon_{i(t+2)}, \ldots\right),
$$

in which case $\gamma_{s} \neq 0$ for all $s$. Finally, if $x_{i t} \vdash\left(\varepsilon_{i(t+j)}, \varepsilon_{i(t+j+1)}, \ldots\right)$ we say that $x_{i t}$ is endogenous but predetermined for $\varepsilon_{i(t+j)}$, and the corresponding $\gamma \mathrm{s}$ vanish.

\subsection{The Order of the Estimation Error}

Let us write the standardized estimation error as

$$
\widehat{\beta}-\beta=\left(\frac{1}{N T} \sum_{t=1}^{T-1} X_{t}^{* \prime} M_{t} X_{t}^{*}\right)^{-1} \frac{1}{N T} \sum_{t=1}^{T-1} X_{t}^{* \prime} M_{t} \varepsilon_{t}^{*} \equiv A_{N T}^{-1} b_{N T} .
$$

A key result is given in the following theorem.

Theorem 3.1. Under Assumption 1 if $x_{i t}$ is predetermined, in the sense that $E\left(x_{i t} \varepsilon_{i(t+j)}\right)=0$ for $j \geq 0$, then

$$
E\left(b_{N T}\right)=O\left(\frac{m}{N}\right) .
$$

If $x_{i t}$ is endogenous, in the sense that $E\left(x_{i t} \varepsilon_{i t}\right) \neq 0$, then

$$
E\left(b_{N T}\right)=O\left(\frac{m T}{N}\right) .
$$

Intuitively, in the predetermined case the "endogeneity bias" vanishes as $T \rightarrow$ $\infty$ because the within-group OLS (WG) estimator is large- $T$ consistent. However, WG is not $T$-consistent in the endogenous explanatory variable case. These results also highlight the impact of $m$ - the dimension of the instrument vector $z_{i t}$ on the order of magnitude of the bias. 


\subsection{The asymptotic bias of GMM}

The previous setup can be used to extend the results in Alvarez and Arellano (2003) to analyzing the asymptotic properties of GMM estimators with general predetermined or endogenous explanatory variables. Here we provide an informal discussion of consistency by drawing parallels with the corresponding AlvarezArellano results.

Let us first consider the probability limit of $(N T)^{-1}\left(X^{* \prime} M X^{*}\right)$. It is useful to introduce at this point the time series individual-specific linear projection of $x_{i t}$ on $\left\{z_{i t}, z_{i(t-1)}, z_{i(t-2)}, \ldots\right\}$ :

$$
p_{i t} \equiv E^{*}\left(x_{i t} \mid z_{i t}, z_{i(t-1)}, \ldots\right)=\phi_{i}+\sum_{j=0}^{\infty} \Phi_{j} z_{i(t-j)} .
$$

Letting $\xi_{i t}$ be the corresponding projection error, we have

$$
x_{i t}=p_{i t}+\xi_{i t}
$$

If $x_{i t}$ is predetermined then $x_{i t}=p_{i t}$ and the errors $\xi_{i t}$ are identically zero for all $i$ and $t$. In such a case, using similar arguments as in Alvarez and Arellano (2003), when $T / N \rightarrow c<\infty$ we can obtain

$$
\operatorname{plim}_{T \rightarrow \infty, N \rightarrow \infty} \frac{1}{N T} X^{* \prime} M X^{*}=E\left[\left(x_{i t}-\mu_{x i}\right)\left(x_{i t}-\mu_{x i}\right)^{\prime}\right]
$$

where $\mu_{x i}$ corresponds to the partition $\mu_{i}=\left(0, \mu_{x i}^{\prime}, \mu_{z i}^{\prime}\right)^{\prime}$.

However, when $x_{i t}$ is endogenous we have

$$
\frac{1}{N T} X^{* \prime} M X^{*}=\frac{1}{N T} P^{* \prime} M P^{*}+\frac{1}{N T} \Xi^{* \prime} M P^{*}+\frac{1}{N T} P^{* \prime} M \Xi^{*}+\frac{1}{N T} \Xi^{* \prime} M \Xi^{*}
$$

where $X^{*}=P^{*}+\Xi^{*}$ denotes the matrix and orthogonal deviation counterpart to $x_{i t}=p_{i t}+\xi_{i t}$. By analogy with the Alvarez-Arellano results, when $T / N \rightarrow c<\infty$ we have

$$
\frac{1}{N T} P^{* \prime} M P^{*} \stackrel{p}{\rightarrow} E\left[\left(p_{i t}-\phi_{i}\right)\left(p_{i t}-\phi_{i}\right)^{\prime}\right]
$$




$$
\begin{gathered}
\frac{1}{N T} P^{* \prime} M \Xi^{*}=O\left(\frac{m}{N}\right) \\
\frac{1}{N T} \Xi^{* \prime} M \Xi^{*}=O\left(\frac{m T}{N}\right) .
\end{gathered}
$$

Decomposition (3.9) can also be used to examine the probability limit of $(N T)^{-1}\left(X^{* \prime} M \varepsilon^{*}\right)$. We have

$$
\operatorname{plim}_{T \rightarrow \infty, N \rightarrow \infty} \frac{1}{N T} X^{* \prime} M \varepsilon^{*}=\operatorname{plim}_{T \rightarrow \infty, N \rightarrow \infty} \frac{1}{N T} P^{* \prime} M \varepsilon^{*}+\operatorname{plim}_{T \rightarrow \infty, N \rightarrow \infty} \frac{1}{N T} \Xi^{* \prime} M \varepsilon^{*} .
$$

Extending the Alvarez-Arellano results (Lemma 2), it turns out that the first term on the right hand side of (3.15) vanishes, but $(N T)^{-1}\left(\Xi^{* \prime} M \varepsilon^{*}\right)$ is of order $O(m T / N)$.

Thus, when $x_{i t}$ is endogenous and $T / N \rightarrow c<\infty$, the form of the asymptotic bias is given by

$$
\begin{aligned}
\operatorname{plim}_{T \rightarrow \infty, N \rightarrow \infty}(\widehat{\beta}-\beta)= & \left\{E\left[\left(p_{i t}-\phi_{i}\right)\left(p_{i t}-\phi_{i}\right)^{\prime}\right]+\operatorname{plim}_{T \rightarrow \infty, N \rightarrow \infty} \frac{1}{N T} \Xi^{* \prime} M \Xi^{*}\right\}^{-1} \\
& \times \operatorname{plim}_{T \rightarrow \infty, N \rightarrow \infty} \frac{1}{N T} \Xi^{* \prime} M \varepsilon^{*}
\end{aligned}
$$

or

$$
\operatorname{plim}_{T \rightarrow \infty, N \rightarrow \infty}(\widehat{\beta}-\beta)=\left\{E\left[\left(p_{i t}-\phi_{i}\right)\left(p_{i t}-\phi_{i}\right)^{\prime}\right]+E\left(\xi_{i t} \xi_{i t}^{\prime}\right) \frac{m c}{2}\right\}^{-1} \gamma_{0} \frac{m c}{2} .
$$

Note that when $x$ is predetermined $\gamma_{0}=0$ and $\xi_{i t} \equiv 0$, so that the bias vanishes as long as $c<\infty$. The conclusion is that GMM is inconsistent in the endogenous explanatory variable situation. In the predetermined case it is consistent, but it can be expected to exhibit a bias in the asymptotic distribution of order $O(m / N)$, similar to those reported in Alvarez and Arellano (2003) for autoregressive models.

The intuition for these results is that in the predetermined case the endogeneity bias tends to disappear as $T$ increases, whereas in the endogenous case it does not. So having an increasing number of moment conditions results in a larger bias when the explanatory variables are endogenous. 


\section{Auxiliary VAR with Individual Effects}

Let us consider a stable $\operatorname{VAR}(1)$ process for an $m \times 1$ vector $z_{i t}$ :

$$
\begin{gathered}
z_{i t}=A z_{i(t-1)}+(I-A) \mu_{i}+v_{i t}(t=1, \ldots, T) \\
E\left(v_{i t} \mid z_{i}^{t-1}, \mu_{i}\right)=0
\end{gathered}
$$

where $z_{i}^{t-1}=\left(z_{i 0}^{\prime}, \ldots, z_{i(t-1)}^{\prime}\right)^{\prime}$, and $\operatorname{Var}\left(v_{i t}\right)=\Omega_{t}$. For notational convenience we assume here that $z_{i 0}$ is also observed.

It is appropriate to conduct our discussion at the multivariate level because typically the conditioning variable will be a vector rather than a scalar, even if we are dealing with a single-equation model. However, we restrict attention to a first-order process for simplicity. Generalization to higher-order processes is cumbersome but straightforward.

If the process for individual $i$ started in the infinite past:

$$
z_{i 0}=\mu_{i}+\sum_{j=0}^{\infty} A^{j} v_{i(-j)}
$$

Since we wish to allow for the possibility that the process started in any given period, and that different individuals started at different times, we treat $\left(z_{i 0}, \mu_{i}\right)$ as realizations of some arbitrary cross-sectional joint distribution.

Let $E\left(\mu_{i}\right)=\mu, \operatorname{Var}\left(\mu_{i}\right)=\Omega_{\mu}$, and let the linear projection of $z_{i 0}$ on $\mu_{i}$ be

$$
z_{i 0}=\tau_{0}+\Upsilon_{1} \mu_{i}+v_{i 0}^{\dagger}
$$

where $E\left(v_{i 0}^{\dagger}\right)=0$ and $\operatorname{Var}\left(v_{i 0}^{\dagger}\right)=\Gamma_{0}$. In general, $\tau_{0}, \Upsilon_{1}$, and $\Gamma_{0}$ are free parameters, but if the process is mean stationary $\tau_{0}=0$, and $\Upsilon_{1}=I_{m}$. If the process is also stationary in variance, $\Omega_{t}=\Omega$ for all $t$, and $\Gamma_{0}=\sum_{j=0}^{\infty} A^{j} \Omega A^{j \prime}$. In any event, $\mu_{i}$ denotes the mean of the steady state distribution of the process for individual $i$, and $\mu$ is the cross-sectional mean of $\mu_{i}$. 
VAR Forecasts For $s>0$ we have

$$
z_{i(t+s)}=\mu_{i}+A^{s}\left(z_{i t}-\mu_{i}\right)+v_{i(t+s)}+A v_{i(t+s-1)}+\ldots+A^{s-1} v_{i(t+1)} .
$$

Therefore,

$$
E_{t}\left(z_{i(t+s)}\right)=E_{t}\left(\mu_{i}\right)+A^{s}\left[z_{i t}-E_{t}\left(\mu_{i}\right)\right]
$$

and

$$
E_{t}\left(z_{i t}^{*}\right)=c_{t}\left[z_{i t}-\frac{1}{T-t} \sum_{s=1}^{T-t} E_{t}\left(z_{i(t+s)}\right)\right]
$$

so that

$$
E_{t}\left(z_{i t}^{*}\right)=c_{t}\left(I-\frac{1}{T-t} \sum_{s=1}^{T-t} A^{s}\right)\left[z_{i t}-E_{t}\left(\mu_{i}\right)\right] .
$$

Notice that $E_{t}\left(z_{i t}^{*}\right)$ only depends on $z_{i t}$ given $E_{t}\left(\mu_{i}\right)$. This is so because we are dealing with a first-order VAR. However, since $E_{t}\left(\mu_{i}\right)$ depends on all lags, so does $E_{t}\left(z_{i t}^{*}\right)$.

Similarly,

$$
E_{t}\left(z_{i(t-j)}^{*}\right)=c_{t}\left\{z_{i(t-j)}-\frac{1}{T-t}\left[z_{i(t-j+1)}+\ldots+z_{i t}+E_{t}\left(z_{i(t+1)}+\ldots+z_{i(T-j)}\right)\right]\right\}
$$

where

$$
E_{t}\left(z_{i(t+1)}+\ldots+z_{i(T-j)}\right)=(T-t-j) E_{t}\left(\mu_{i}\right)+\left(\sum_{s=1}^{T-t-j} A^{s}\right)\left[z_{i t}-E_{t}\left(\mu_{i}\right)\right] .
$$

The same is true if conditional expectations $E_{t}($.$) are replaced by linear pro-$ jections $E_{t}^{*}($.$) . The form of E_{t}^{*}\left(\mu_{i}\right)$ is given in the following Theorem.

Theorem 4.1. (Sequential linear projections of the effects) For the VAR(1) model presented above, the linear projection of the vector of individual effects $\mu_{i}$ on $z_{i}^{t}$ is given by the following recursive updating formula for $t \geq 0$ :

$$
E_{t}^{*}\left(\mu_{i}\right)=\mathcal{H}_{t}^{-1} \delta_{i t}
$$


where

$$
\begin{array}{r}
\mathcal{H}_{0}=I+\Omega_{\mu} \Upsilon_{1}^{\prime} \Gamma_{0}^{-1} \Upsilon_{1} \\
\delta_{i 0}=\mu+\Omega_{\mu} \Upsilon_{1}^{\prime} \Gamma_{0}^{-1}\left(z_{i 0}-\tau_{0}\right)
\end{array}
$$

and for $t \geq 1$ :

$$
\begin{array}{r}
\mathcal{H}_{t}=\mathcal{H}_{t-1}+\Omega_{\mu}(I-A)^{\prime} \Omega_{t}^{-1}(I-A) \\
\delta_{i t}=\delta_{i(t-1)}+\Omega_{\mu}(I-A)^{\prime} \Omega_{t}^{-1}\left(z_{i t}-A z_{i(t-1)}\right) .
\end{array}
$$

For the stationary case, by inserting $\tau_{0}=0, \Upsilon_{1}=I_{m}$, and $\Omega_{t}=\Omega$ for all $t$ in the result given in the Theorem, we get:

$$
E_{t}^{*}\left(\mu_{i}\right)=\left[I+\Lambda_{0}+t \Lambda_{1}(I-A)\right]^{-1}\left[\mu+\Lambda_{0} z_{i 0}+\Lambda_{1} \sum_{s=1}^{t}\left(z_{i s}-A z_{i(s-1)}\right)\right] .
$$

where $\Lambda_{0}=\Omega_{\mu} \Gamma_{0}^{-1}$ and $\Lambda_{1}=\Omega_{\mu}(I-A)^{\prime} \Omega^{-1}$ (further details on the stationary case are in the Appendix).

Note that the linear projection of the vector of individual effects in a $\operatorname{VAR}(1)$ model with mean stationarity and constant $\Omega$ but no covariance stationarity is of the same form as the result in (4.15), but treating $\Gamma_{0}$ as an unrestricted covariance matrix.

Another intermediate possibility is one in which $\Omega_{t}=\Omega$ for all $t$, but initial conditions are left fully unrestricted, so that $\tau_{0}, \Upsilon_{1}$, and $\Gamma_{0}$ are treated as free parameters. This is a case of special interest because $E_{t}^{*}\left(\mu_{i}\right)$ depends on a fixed number of parameters which does not increase with $t$. 


\section{The Form of the Optimal Instruments}

An expression for the optimal instruments is given by ${ }^{4}$

$$
\begin{aligned}
E_{t}\left(x_{i t}^{*}\right) & =B_{0} E_{t}\left(z_{i t}^{*}\right)+B_{1} E_{t}\left(z_{i(t-1)}^{*}\right)+\ldots+B_{q} E_{t}\left(z_{i(t-q)}^{*}\right) \\
& =B^{\dagger} E_{t}\left(z_{i t(q)}^{*}\right)
\end{aligned}
$$

where $B^{\dagger}=\left(B_{0}, \ldots, B_{q}\right)$ and $z_{i t(q)}=\left(z_{i t}^{\prime}, \ldots, z_{i(t-q)}^{\prime}\right)^{\prime}$.

If all $x$ 's are predetermined for some lead of $\varepsilon$, the form of $E_{t}\left(x_{i t}^{*}\right)$ and $B^{\dagger}$ is known given knowledge of the parameters of the VAR for $z_{i t}$, so that estimation of $\beta$ can be based on the sample moments

$$
\sum_{i} \sum_{t} \widehat{E}_{t}\left(x_{i t}^{*}\right)\left(y_{i t}^{*}-x_{i t}^{* \prime} \beta\right)
$$

where $\widehat{E}_{t}\left(x_{i t}^{*}\right)$ is an estimate of $E_{t}\left(x_{i t}^{*}\right)$. Since the optimal instrument has the same dimension as $\beta$ no further weighting of the moments is required.

If on the other hand part of the $x$ 's are completely endogenous, then $q$ and some of the elements of $B^{\dagger}$ are unknown. Thus, we consider GMM estimates of $\beta$ based on the moments

$$
\sum_{i} \sum_{t} \widehat{E}_{t}\left(z_{i t(q)}^{*}\right)\left(y_{i t}^{*}-x_{i t}^{* \prime} \beta\right)
$$

In this case the instruments may have a larger dimension than $\beta$ so that weighting of the moments may be required. Indeed, the choice of weight matrix here is equivalent to choosing an estimation method for $B^{\dagger}$. A suggested weighting is discussed below. ${ }^{5}$ Next, we consider some examples.

\footnotetext{
${ }^{4}$ Expression (5.1) is saying that

$$
E\left(x_{i t}^{*}-B_{0} z_{i t}^{*}-B_{1} z_{i(t-1)}^{*}-\ldots-B_{q} z_{i(t-q)}^{*} \mid z_{i}^{t}\right)=0
$$

or that the time-series individual-specific projection of $x_{i t}$ on $z_{i}^{t}$ only depends on the first $q$ lags of $z_{i t}$ and satisfies $E\left(x_{i t}-B_{0} z_{i t}-B_{1} z_{i(t-1)}-\ldots-B_{q} z_{i(t-q)}-\xi_{i} \mid z_{i}^{t}\right)=0$.

${ }^{5}$ Note that estimation of an extended VAR for $\left(x_{i t}^{\prime}, z_{i t}^{\prime}\right)$ subject to exclusion restrictions (of
} 
Example 1: An Equation from a VAR We have

$$
\begin{gathered}
w_{1 i t}=a_{1}^{\prime} w_{i(t-1)}+\eta_{i}+\varepsilon_{i t} \\
E\left(\varepsilon_{i t} \mid w_{i}^{t-1}\right)=0 .
\end{gathered}
$$

In this case $z_{i t}=w_{i(t-1)}$ and $x_{i t}=z_{i t}$, so that $B_{0}=I$, and $B_{j}=0$ for $j>0$.

When the model of interest is a VAR, the auxiliary and substantive models coincide, except for the fact that the auxiliary model is based on stronger assumptions than the substantive model. In contrast, in conditional models the auxiliary assumptions give a parametric form to the feedback processes, which in the substantive model will typically remain unspecified. ${ }^{6}$

Example 2: Partial Adjustment Regression We have

$$
\begin{gathered}
y_{i t}=\alpha y_{i(t-1)}+\beta_{0} w_{i t}+\beta_{1} w_{i(t-1)}+\eta_{i}+\varepsilon_{i t} \\
E\left(\varepsilon_{i t} \mid y_{i}^{t-1}, w_{i}^{t}\right)=0 .
\end{gathered}
$$

In this case $z_{i t}=\left(y_{i(t-1)}, w_{i t}\right)^{\prime}, x_{i t}=\left(y_{i(t-1)}, w_{i t}, w_{i(t-1)}\right)^{\prime}$,

$$
x_{i t}=\left(\begin{array}{c}
y_{i(t-1)} \\
w_{i t} \\
w_{i(t-1)}
\end{array}\right)=\left(\begin{array}{ll}
1 & 0 \\
0 & 1 \\
0 & 0
\end{array}\right) z_{i t}+\left(\begin{array}{ll}
0 & 0 \\
0 & 0 \\
0 & 1
\end{array}\right) z_{i(t-1)} \equiv B_{0} z_{i t}+B_{1} z_{i(t-1)} .
$$

Example 3: Cross-Country Growth The next example is an augmented Solow model of the determinants of growth as in Caselli, Esquivel, and Lefort (1996). The equation is

$$
y_{i t}=\alpha y_{i(t-1)}+s_{i(t-1)} \gamma+f_{i(t-1)}^{\prime} \delta+\eta_{i}+\varepsilon_{i t}
$$

lagged $x^{\prime} \mathrm{s}$ ) is not warranted. The reason is that the $B^{\dagger}$ are meant to be the coefficients of a linear projection of $x_{i t}$ on $z_{i}^{t}$ and individual effects, and there is no reason why the errors in this projection should be serially uncorrelated.

${ }^{6}$ Optimal instruments for scalar autoregressive models are discussed in Appendix C. 


$$
E\left(\varepsilon_{i t} \mid y_{i}^{t-1}, s_{i}^{t-1}, f_{i}^{t-2}\right)=0 .
$$

The time interval is 5 years, $y_{i t}$ is log per-capita GDP, $f_{i(t-1)}$ is a vector of flow variables containing the rates of investment and population growth, and $s_{i(t-1)}$ is a stock variable measuring the secondary school enrollment rate.

In this case $z_{i t}=\left(y_{i(t-1)}, s_{i(t-1)}, f_{i(t-2)}^{\prime}\right)^{\prime}$, and

$$
\left(\begin{array}{c}
y_{i(t-1)} \\
s_{i(t-1)} \\
f_{i(t-1)}
\end{array}\right)=\left(\begin{array}{ccc}
1 & 0 & 0 \\
0 & 1 & 0 \\
A_{31} & A_{32} & A_{33}
\end{array}\right)\left(\begin{array}{c}
y_{i(t-1)} \\
s_{i(t-1)} \\
f_{i(t-2)}
\end{array}\right)+\left(\begin{array}{c}
0 \\
0 \\
u_{f i(t-1)}
\end{array}\right)
$$

where the last equation coincides with the equation for $f_{i(t-2)}$ in the VAR model of $z_{i t}$. Thus,

$$
E_{t}\left(x_{i t}^{*}\right)=B_{0} E_{t}\left(z_{i t}^{*}\right)
$$

Since $B_{0}$ is in general a squared, nonsingular matrix, it is irrelevant in the construction of optimal instruments, and the optimal instrument in the growth example can be simply taken to be $E\left(z_{i t}^{*} \mid z_{i}^{t}\right)$. So in both the VAR and growth examples we have $B_{0}=I$ and $B_{j}=0$ for $j>0$.

Example 4: Euler Equation for Household Consumption The last example is an Euler equation of the type considered by Zeldes (1989) and others:

$$
\ln \left(1+r_{i t}\right)=\alpha \Delta \ln c_{i t}+\beta^{\prime} w_{i t}+\eta_{i}+\varepsilon_{i t}
$$

where $w_{i t}$ is a vector of changes in family size variables, $r_{i t}$ is the rate of return on a riskless asset, and $c_{i t}$ is consumption; $\eta_{i}$ captures heterogeneity in discount rates, and $\varepsilon_{i t}$ unobservable changes in tastes and expectation errors.

The vector of instruments $z_{i t}$ contains $w_{i t}$, lagged income, and marginal taxes, so that both returns and consumption growth are treated as completely endogenous variables. 
In this case, letting $z_{i t}=\left(w_{i t}^{\prime}, z_{2 i t}^{\prime}\right)^{\prime}$, we have

$$
\begin{aligned}
\Delta \ln c_{i t} & =\sum_{j=0}^{q} \pi_{j}^{\prime} z_{i(t-j)}+\zeta_{i}+\xi_{i t} \\
w_{i t} & =(I, 0) z_{i t}
\end{aligned}
$$

or

$$
E_{t}\left(\begin{array}{c}
\left(\Delta \ln c_{i t}\right)^{*} \\
w_{i t}^{*}
\end{array}\right)=\left(\begin{array}{c}
\pi_{0}^{\prime} \\
I, 0
\end{array}\right) E_{t}\left(z_{i t}^{*}\right)+. .+\left(\begin{array}{c}
\pi_{q}^{\prime} \\
0
\end{array}\right) E_{t}\left(z_{i(t-J)}^{*}\right)
$$

Thus, there is a nontrivial specification of the $\left(B_{0} \ldots B_{q}\right)$ due to the presence of completely endogenous variables and external instruments.

\section{PML estimation of the VAR model}

To obtain feasible estimators, the coefficients parameterizing the optimal instruments must be replaced by sample estimates. The suggested estimates are Gaussian pseudo-maximum likelihood statistics of the VAR auxiliary model. The estimates are obtained under the assumptions that the data are normally distributed and the error variance matrices are homoskedastic, but the multivariate linear projection of the initial observations on the effects is left unrestricted. Estimates of this type were considered by Blundell and Smith (1991), and Alvarez and Arellano (2003) for a scalar autoregressive model. ${ }^{7}$ Alvarez and Arellano obtained a useful concentrated likelihood that only depended on the autoregressive parameter, and found that the maximizer of this criterion behaved very well in simulations. ${ }^{8}$ This section reports a multivariate generalization of the PML

\footnotetext{
${ }^{7}$ Blundell and Smith (1991) considered a generalized least squares estimator of the same model, which has been further discussed by Blundell and Bond (1998).

${ }^{8}$ This is the PML estimate that does not restrict the individual effect variance to be nonnegative. Alvarez and Arellano also discussed an alternative PML that enforced non-negativity, but in such a case a boundary solution may occur.
} 
results of Alvarez and Arellano, which will be required in practice for obtaining feasible optimal instruments. ${ }^{9}$

As shown in the Appendix, the log-likelihood given $z_{i 0}$ (under homoskedasticity and a joint normal distribution for $\mu_{i}$ and $z_{i 0}$ with unrestricted mean and covariance matrix) can be written as:

$$
\begin{aligned}
& \ln f\left(z_{i 1}, \ldots, z_{i T} \mid z_{i 0}\right)=-\frac{(T-1)}{2} \ln \operatorname{det} \Omega-\frac{1}{2} \sum_{t=1}^{T-1} u_{i t}^{* \prime} \Omega^{-1} u_{i t}^{*} \\
& -\frac{1}{2} \ln \operatorname{det} \Theta_{0}-\frac{1}{2}\left(\bar{u}_{i}-\phi_{0}-\Phi_{1} z_{i 0}\right)^{\prime} \Theta_{0}^{-1}\left(\bar{u}_{i}-\phi_{0}-\Phi_{1} z_{i 0}\right) .
\end{aligned}
$$

where $u_{i t}^{*}=z_{i t}^{*}-A z_{i(t-1)}^{*}, \bar{u}_{i}=\bar{z}_{i}-A \bar{z}_{i(-1)}$ and

$$
\bar{u}_{i} \mid z_{i 0} \sim \mathcal{N}\left(\phi_{0}+\Phi_{1} z_{i 0}, \Theta_{0}\right)
$$

Concentrating $\phi_{0}, \Phi_{1}, \Theta_{0}$ and $\Omega$, the PMLE of $A$ solves

$$
\begin{aligned}
\widetilde{A}= & \arg \min \left\{\ln \operatorname{det}\left(Z^{* \prime}-A Z_{-1}^{* \prime}\right)\left(Z^{*}-Z_{-1}^{*} A^{\prime}\right)\right. \\
& \left.+\frac{1}{T-1} \ln \operatorname{det}\left(\bar{Z}^{\prime}-A \bar{Z}_{-1}^{\prime}\right) S_{0}\left(\bar{Z}-\bar{Z}_{-1} A^{\prime}\right)\right\}
\end{aligned}
$$

where $S_{0}=I-F\left(F^{\prime} F\right)^{-1} F^{\prime}$ and $F$ is the $N \times(m+1)$ matrix of constants and initial observations $f_{i 0}=\left(1, z_{i 0}^{\prime}\right)^{\prime}$.

PML estimates of the remaining parameters Given $\widetilde{A}$, they are given by

$$
\begin{gathered}
\widetilde{\Omega}=\frac{1}{N(T-1)} \sum_{i=1}^{N} \sum_{t=1}^{T-1}\left(z_{i t}^{*}-\widetilde{A} z_{i(t-1)}^{*}\right)\left(z_{i t}^{*}-\widetilde{A} z_{i(t-1)}^{*}\right)^{\prime} \\
\widetilde{\Phi}=\left(\widetilde{\phi}_{0}, \widetilde{\Phi}_{1}\right)=\left(\bar{Z}^{\prime}-\widetilde{A} \bar{Z}_{-1}^{\prime}\right) F\left(F^{\prime} F\right)^{-1}
\end{gathered}
$$

\footnotetext{
${ }^{9}$ One problem with this method as an estimator of substantive VAR parameters is that it is not robust to lack of time series homoskedasticity.
} 
and letting $\widetilde{\bar{u}_{i}}=\bar{z}_{i}-\widetilde{A} \bar{z}_{i(-1)}$ :

$$
\widetilde{\Theta}_{0}=\frac{1}{N} \sum_{i=1}^{N}\left(\widetilde{\bar{u}}_{i}-\widetilde{\phi}_{0}-\widetilde{\Phi}_{1} z_{i 0}\right)\left(\widetilde{\bar{u}}_{i}-\widetilde{\phi}_{0}-\widetilde{\Phi}_{1} z_{i 0}\right)^{\prime} .
$$

The estimated mean and variance matrix of $\eta_{i}=(I-A) \mu_{i}$ can be obtained as:

$$
\begin{gathered}
\widetilde{\Omega}_{\eta}=\widetilde{\Theta}_{0}+\widetilde{\Phi}_{1} \widetilde{\Sigma}_{0} \widetilde{\Phi}_{1}^{\prime}-\widetilde{\Omega} / T \\
\widetilde{\eta}=\widetilde{\phi}_{0}+\widetilde{\Phi}_{1} \bar{z}_{0}
\end{gathered}
$$

where $\bar{w}_{0}$ and $\widetilde{\Sigma}_{0}$ are the sample mean and variance of $w_{i 0}$.

Finally, estimates of $\Upsilon_{1}$ and $\tau_{0}$ are given by

$$
\begin{gathered}
\widetilde{\Upsilon}_{1}=\widetilde{\Sigma}_{0} \widetilde{\Phi}_{1}^{\prime} \widetilde{\Omega}_{\eta}^{-1}(I-\widetilde{A}) \\
\widetilde{\tau}_{0}=\bar{z}_{0}-\widetilde{\Sigma}_{0} \widetilde{\Phi}_{1}^{\prime} \widetilde{\Omega}_{\eta}^{-1} \widetilde{\eta}
\end{gathered}
$$

\section{Inference with Feasible SIV Estimators}

An unfeasible estimator for a parameterization of the instruments takes the form

$$
\widehat{\beta}_{U F}=\left(\sum_{i=1}^{N} \sum_{t=1}^{T-1} h_{t}\left(z_{i}^{t}, \gamma\right) x_{i t}^{* \prime}\right)^{-1}\left(\sum_{i=1}^{N} \sum_{t=1}^{T-1} h_{t}\left(z_{i}^{t}, \gamma\right) y_{i t}^{*}\right),
$$

where $\gamma$ is a pseudo true value that in practice will be defined by the probability limit of some sample statistic.

Under standard regularity conditions, for fixed $T$ and large $N$,

$$
\sqrt{N(T-1)}\left(\widehat{\beta}_{U F}-\beta\right) \stackrel{d}{\rightarrow} \mathcal{N}\left(0, V_{U F}\right)
$$

with asymptotic variance matrix given by

$$
V_{U F}=(T-1)\left[E\left(H_{i}^{\prime} X_{i}^{*}\right)\right]^{-1} E\left(H_{i}^{\prime} \varepsilon_{i}^{*} \varepsilon_{i}^{* \prime} H_{i}\right)\left[E\left(X_{i}^{* \prime} H_{i}\right)\right]^{-1}
$$


where $H_{i}=\left(h_{i 1}, \ldots, h_{i(T-1)}\right)^{\prime}, h_{i t}=h_{t}\left(z_{i}^{t}, \gamma\right), X_{i}^{*}=\left(x_{i 1}^{*}, \ldots, x_{i(T-1)}^{*}\right)^{\prime}$, and $\varepsilon_{i}^{*}=$ $\left(\varepsilon_{i 1}^{*}, \ldots, \varepsilon_{i(T-1)}^{*}\right)^{\prime}$.

Provided $E_{t}\left(\varepsilon_{i t}^{2}\right)=\sigma^{2}$ and $E_{t+j}\left(\varepsilon_{i t} \varepsilon_{i(t+j)}\right)=0$ for $j>0$ it will be the case that

$$
V_{U F}=\sigma^{2}(T-1)\left[E\left(H_{i}^{\prime} X_{i}^{*}\right)\right]^{-1} E\left(H_{i}^{\prime} H_{i}\right)\left[E\left(X_{i}^{* \prime} H_{i}\right)\right]^{-1} .
$$

If in addition $E_{t}\left(x_{i t}^{*}\right)=h_{t}\left(z_{i}^{t}, \gamma\right)$ then $V_{U F}$ coincides with the variance bound.

A feasible estimator $\widehat{\beta}_{F}$ is of the same form as $\widehat{\beta}_{U F}$ but $\gamma$ is replaced by an estimate $\widehat{\gamma}$. Provided, $p \lim _{N \rightarrow \infty} \widehat{\gamma}=\gamma$ and $\sqrt{N}(\widehat{\gamma}-\gamma)$ is bounded in probability, the feasible and unfeasible estimators are asymptotically equivalent.

To see this first note that

$$
\sqrt{N}\left(\widehat{\beta}_{F}-\beta\right)=\left(\frac{1}{N} \sum_{i=1}^{N} \sum_{t=1}^{T-1} h_{t}\left(z_{i}^{t}, \gamma\right) x_{i t}^{* \prime}\right)^{-1} \frac{1}{\sqrt{N}} \sum_{i=1}^{N} \sum_{t=1}^{T-1} h_{t}\left(z_{i}^{t}, \widehat{\gamma}\right) v_{i t}^{*}+o_{p}(1) .
$$

Moreover, using the mean value theorem

$$
\begin{aligned}
\frac{1}{\sqrt{N}} \sum_{i=1}^{N} \sum_{t=1}^{T-1} h_{t}\left(z_{i}^{t}, \widehat{\gamma}\right) v_{i t}^{*}= & \frac{1}{\sqrt{N}} \sum_{i=1}^{N} \sum_{t=1}^{T-1} h_{t}\left(z_{i}^{t}, \gamma\right) v_{i t}^{*} \\
& +\left(\frac{1}{N} \sum_{i=1}^{N} \sum_{t=1}^{T-1} \frac{\partial h_{t}\left(z_{i}^{t}, \gamma\right)}{\partial \gamma^{\prime}} v_{i t}^{*}\right) \sqrt{N}(\widehat{\gamma}-\gamma)+o_{p}(1)
\end{aligned}
$$

Since $E\left(v_{i t}^{*} \mid z_{i}^{t}\right)=0$, we have that $p \lim _{N \rightarrow \infty} N^{-1} \sum_{i=1}^{N} \sum_{t=1}^{T-1} v_{i t}^{*} \partial h_{t}\left(z_{i}^{t}, \gamma\right) / \partial \gamma^{\prime}=$ 0 . Therefore, the second term on the rhs of the previous expression is $o_{p}(1)$. From this it follows that $\sqrt{N}\left(\widehat{\beta}_{F}-\beta\right) \stackrel{d}{\rightarrow} \mathcal{N}\left(0, V_{U F}\right)$. Similar arguments can be used for the cases where $T$ or both $N$ and $T$ tend to infinity.

Consistent Estimation of the Asymptotic Variance Matrix A natural estimate of the "Hessian" component of $V_{U F}$ is

$$
\widehat{\Psi}_{N T}=\frac{1}{N(T-1)} \sum_{i=1}^{N} \sum_{t=1}^{T-1} \widehat{h}_{i t} x_{i t}^{* \prime}
$$


where $\widehat{h}_{i t}=h_{t}\left(z_{i}^{t}, \widehat{\gamma}\right) . \widehat{\Psi}_{N T}$ is an $N$-consistent estimator of $(T-1)^{-1} E\left(H_{i}^{\prime} X_{i}^{*}\right)$, but also an $N$ and $T$ consistent estimator of $p \lim _{T \rightarrow \infty, N \rightarrow \infty} \widehat{\Psi}_{N T}$.

The conventional robust fixed- $T$ estimate of the "outer-product" component of $V_{U F}$ is

$$
\widetilde{\Upsilon}_{N T}=\frac{1}{N(T-1)} \sum_{i=1}^{N} \widehat{H}_{i}^{\prime} \widehat{\varepsilon}_{i}^{*} \widehat{\varepsilon}_{i}^{* \prime} \widehat{H}_{i}
$$

where $\widehat{\varepsilon}_{i t}^{*}=y_{i t}^{*}-x_{i t}^{* \prime} \widehat{\beta}_{F}$ and $\widehat{H}_{i}$ contains the estimated instruments $\widehat{h}_{i t}$.

It can be shown that

$$
\widetilde{\Upsilon}_{N T}=\widehat{\Omega}_{0}+\sum_{\ell=1}^{T-2}\left(1-\frac{\ell}{T-1}\right)\left(\widehat{\Omega}_{\ell}+\widehat{\Omega}_{\ell}^{\prime}\right)
$$

where

$$
\widehat{\Omega}_{\ell}=\frac{1}{N(T-1-\ell)} \sum_{i=1}^{N} \sum_{t=\ell+1}^{T-1} \widehat{\varepsilon}_{i t}^{*} \widehat{\varepsilon}_{i(t-\ell)}^{*} \widehat{h}_{i t} \widehat{h}_{i(t-\ell)}^{\prime}
$$

for $\ell=0,1, \ldots, T-2 .^{10}$

From a non-fixed $T$ perspective, we consider the following estimator of the outer-product component:

$$
\widehat{\Upsilon}_{N T}=\widehat{\Omega}_{0}+\sum_{\ell=1}^{r}\left(1-\frac{\ell}{r+1}\right)\left(\widehat{\Omega}_{\ell}+\widehat{\Omega}_{\ell}^{\prime}\right) .
$$

where $r \leq T-2$.

For $r=T-2, \widehat{\Upsilon}_{N T}$ particularizes to the standard robust fixed- $T$ formula. In a fixed $T$ context, it is natural to use $\widetilde{\Upsilon}_{N T}$, since the number of $\Omega_{i \ell}$ terms is fixed, and large $N$ ensures consistent estimation of all of them, including $\Omega_{i(T-2)}$, which

\footnotetext{
${ }^{10}$ Note that

$$
\widehat{H}_{i}^{\prime} \widehat{\varepsilon}_{i}^{*} \widehat{\varepsilon}_{i}^{* \prime} \widehat{H}_{i}=\sum_{t=1}^{T-1} \sum_{s=1}^{T-1} \widehat{\varepsilon}_{i t}^{*} \widehat{\varepsilon}_{i s}^{*} \widehat{h}_{i t} \widehat{h}_{i s}^{\prime}=(T-1) \Omega_{i 0}+\sum_{\ell=1}^{T-2}(T-1-\ell)\left(\Omega_{i \ell}+\Omega_{i \ell}^{\prime}\right)
$$

where $\Omega_{i \ell}=(T-1-\ell)^{-1} \sum_{t=\ell+1}^{T-1} \widehat{\varepsilon}_{i t}^{*} \widehat{\varepsilon}_{i(t-\ell)}^{*} \widehat{h}_{i t} \widehat{h}_{i(t-\ell)}^{\prime}$ and $\widehat{\Omega}_{\ell}=N^{-1} \sum_{i=1}^{N} \Omega_{i \ell}$ for $\ell=$ $0,1, \ldots, T-2$.
} 
is a time series average with a single observation. However, from a non-fixed $T$ perspective, the bound $r$ on the number of autocovariances used to form $\widehat{\Upsilon}_{N T}$ should be chosen as a suitable function of $T$ to ensure consistent estimation for a given asymptotic arrangement in $N$ and $T$.

Formula (7.9) is a panel data version of the estimator proposed in Newey and West (1987) for time series data. It has the attractive feature of providing a positive semidefinite estimator for any value of $r .^{11}$

In the time series context, the term $[1-\ell /(r+1)]$ appearing in $(7.9)$ is motivated as a damping factor for reducing the sampling error induced by higher-order sample covariances in the truncated estimate (called modified Bartlett weights in Anderson,1971, 511-513). It is interesting that these weights appear naturally as a feature of the sample covariance formula (7.7) from a cross-sectional perspective.

In the fixed- $T$ panel data context, formula (7.6) is often used with instrument matrices whose number of columns is of order $T$ or $T^{2}$, so that the dimension of $\widetilde{\Upsilon}_{N T}$ itself increases with $T$. Here, however, we are considering optimal instruments $h_{i t}$ with a fixed dimension for any value of $T$.

The proposal is, therefore, to base inference on the following estimate of the asymptotic covariance matrix of $\sqrt{N(T-1)}\left(\widehat{\beta}_{F}-\beta\right)$ for a chosen value of $r$ :

$$
\widehat{V}_{U F}^{\dagger}=\widehat{\Psi}_{N T}^{-1}\left[\widehat{\Omega}_{0}+\sum_{\ell=1}^{r}\left(1-\frac{\ell}{r+1}\right)\left(\widehat{\Omega}_{\ell}+\widehat{\Omega}_{\ell}^{\prime}\right)\right] \widehat{\Psi}_{N T}^{\prime-1}
$$

\section{Optimal Instruments with Completely Endogenous Explanatory Vari-} ables: A Two-Step GMM Method Now we can pursue the discussion on estimation when part of the $x$ 's are completely endogenous and the instruments have a larger dimension than $\beta$. We suggest using the inverse of $\widehat{\Upsilon}_{N T}$ as the weight

\footnotetext{
${ }^{11}$ The estimator in (7.9) relies on cross-sectional independence. A related formula for large $T$, fixed $N$ within-group standard errors that allow for arbitrary cross-sectional dependence is proposed in Arellano (2003).
} 
matrix. Thus, we consider estimators of the form

$$
\begin{aligned}
\widetilde{\beta}= & {\left[\left(\sum_{i} \sum_{t} x_{i t}^{*} \widehat{h}_{i t}^{\prime}\right) \widehat{\Upsilon}_{N T}^{-1}\left(\sum_{i} \sum_{t} \widehat{h}_{i t} x_{i t}^{* \prime}\right)\right]^{-1} } \\
& \left(\sum_{i} \sum_{t} x_{i t}^{*} \widehat{h}_{i t}^{\prime}\right) \widehat{\Upsilon}_{N T}^{-1}\left(\sum_{i} \sum_{t} \widehat{h}_{i t} y_{i t}^{*}\right)
\end{aligned}
$$

where $\widehat{h}_{i t}$ is an estimate of $E_{t}\left(z_{i t(q)}^{*}\right)$ as introduced in (5.3).

Note that $\widetilde{\beta}$ can be regarded as the SIV estimator that uses the following moments that have the same dimension of $\beta$ :

$$
\sum_{i} \sum_{t} \widehat{x}_{i t}^{\dagger}\left(y_{i t}^{*}-x_{i t}^{* \prime} \tilde{\beta}\right)=0
$$

where $\widehat{x}_{i t}^{\dagger}=\left(\sum_{i} \sum_{t} x_{i t}^{*} \widehat{h}_{i t}^{\prime}\right) \widehat{\Upsilon}_{N T}^{-1} \widehat{h}_{i t}$.

\section{Empirical Illustrations}

\subsection{VAR for Firm Panel Data}

We estimate autoregressive employment and wage equations from firm panel data. We first consider the dataset used by Alonso-Borrego and Arellano (1999). This is a balanced panel of 738 Spanish manufacturing companies, for which there are available annual observations for 1983-1990. Secondly we consider a longer panel of 385 firms from the same source (Bank of Spain, Central de Balances) on which 14 years of data are available, also starting in 1983. The average size of the firms in the second panel is more than twice as large as that of those in the first one.

We estimate a first-order VAR model for the logs of employment and wages, denoted $n_{i t}$ and $w_{i t}$ respectively. Individual and time effects are included in both equations. Time effects are removed prior to estimation by taking data in deviations from period-specific cross-sectional means. 
Within-groups (WG), GMM, PML, and projection-restricted simple IV (SIV) estimates are reported. In this illustration PML appears in two different roles. First, it is an input in calculating feasible optimal instruments for SIV estimation. Second, since the auxiliary and substantive models coincide in this illustration, PML can be also regarded as an estimator of the parameters of interest that imposes time-series homoskedasticity. ${ }^{12}$

Table 1 shows the results. Focusing on the leading coefficient in the employment equation, first notice the larger size of the within-group estimates from the longer panel, which is to be expected since WG is known to have a small- $T$ downward bias (Nickell, 1981). Next, the discrepancy between GMM and PML estimates is as noticeable as that between WG and GMM. Finally, SIV estimates are in between GMM and PML. The difference between GMM and PML may be due to finite-sample bias in GMM, heteroskedasticity bias in PML, or a combination of both. SIV estimates are as robust as GMM under a fixed- $T$ asymptotics, but less prone to bias in a double asymptotics. In particular, SIV is robust to time-series heteroskedasticity in short panels whereas PML is not. Reported SIV estimates are half way between GMM and PML.

\subsection{Monte Carlo Simulations}

Next we performed a simulation exercise loosely calibrated to the previous firm panel dataset. The design was chosen from the partial adjustment representation as follows:

$$
\begin{aligned}
& y_{i t}=1+0.8 y_{i(t-1)}-0.5 x_{i t}+0.3 x_{i(t-1)}+\eta_{i}+v_{i t} \\
& x_{i t}=0.5+0.3 x_{i(t-1)}+\xi_{i}+\varepsilon_{i t},
\end{aligned}
$$

\footnotetext{
${ }^{12}$ The PML estimates that we report use unrestricted initial conditions. According to estimates of $\Upsilon_{1}$ for the two panels (reported in Table 1 ) there is some evidence of nonstationary initial conditions.
} 
where all unobservables are iid normally distributed (over both $i$ and $t$ ) with zero mean and $\sigma_{v}^{2}=\sigma_{\varepsilon}^{2}=0.01, \operatorname{Corr}\left(v_{i t}, \varepsilon_{i t}\right)=0, \sigma_{\eta}^{2}=\sigma_{\xi}^{2}=0.09$, and $\operatorname{Corr}\left(\eta_{i}, \xi_{i}\right)=$ 0.6. Therefore, the variance of the fixed effects is 9 times that of the random errors. There is no feedback from lagged $y$ into $x$, and the long run effect of $x$ on $y$ is unity. Initial observations are generated from the stationary distribution of the process. The corresponding VAR is

$$
\left(\begin{array}{l}
y_{i t} \\
x_{i t}
\end{array}\right)=\left(\begin{array}{c}
0.75 \\
0.5
\end{array}\right)+\left(\begin{array}{cc}
0.8 & 0.15 \\
0 & 0.3
\end{array}\right)\left(\begin{array}{l}
y_{i(t-1)} \\
x_{i(t-1)}
\end{array}\right)+\left(\begin{array}{c}
c_{i}+e_{i t} \\
\xi_{i}+\varepsilon_{i t}
\end{array}\right)
$$

where $e_{i t}=v_{i t}-0.5 \varepsilon_{i t}, c_{i}=\eta_{i}-0.5 \xi_{i}$,

$$
\operatorname{Var}\left(\begin{array}{l}
e_{i t} \\
\varepsilon_{i t}
\end{array}\right)=\Omega=\left(\begin{array}{cc}
0.0125 & -0.005 \\
-0.005 & 0.01
\end{array}\right)
$$

and

$$
\operatorname{Var}\left(\begin{array}{c}
c_{i} \\
\xi_{i}
\end{array}\right)=\Omega_{\eta}=\left(\begin{array}{cc}
0.0585 & 0.009 \\
0.009 & 0.09
\end{array}\right)
$$

The implied correlations are $\operatorname{Corr}\left(e_{i t}, \varepsilon_{i t}\right)=-0.447$ and $\operatorname{Corr}\left(c_{i}, \xi_{i}\right)=0.124$.

In Table 2 we report medians and median absolute errors of the WG, GMM, PML, and SIV estimators for $\{N=738, T=8\}$ and $\{N=385, T=14\}$.

In another experiment with $N=738, T=8$ we generated data with trending variances. The specification in this case was

$$
\sigma_{v t}^{2}=\sigma_{\varepsilon t}^{2}=0.01+0.001 t
$$

with $t=0$ selected in such a way that the resulting sequence of variances ranged from .005 to .012. This is also in Table 2. Finally, in Table 3 we report further simulations for $\{N=200, T=8\},\{N=100, T=6\}$, and $\{N=50, T=15\}$. For all cases we conducted 1000 replications.

Focusing again on the leading coefficient in the first equation ( $a_{11}$ with true value of 0.8), Table 2 shows that GMM is downward biased but PML and SIV are virtually median unbiased. The robustness of SIV comes at the cost of a larger 
median absolute error than PML. The third panel of Table 2 for the experiment with trending variance shows that PML is upward biased, which goes in the direction of the empirical findings. Table 3 reports results for other sample sizes. Those in the first two experiments are similar to the Arellano-Bond firm-level data, and the cross-country panels used in growth studies, respectively. The last one illustrates the situation in a smaller panel that would be difficult to classify as either long $T$ or large $N$, small $T$. In all cases SIV is unbiased and has reasonable median absolute errors, which is in contrast to some spectacular GMM biases.

\subsection{Estimating Country Growth Convergence Rates}

Using panel GMM, Caselli, Esquivel, and Lefort (1996) found a surprisingly large estimate of the convergence rate of about 10 percent. This was in sharp contrast with earlier cross-sectional estimates of Barro and Sala-i-Martin, who found convergence rates of 2-3 percent. ${ }^{13}$ Caselli et al. claimed that earlier estimates were biased due to lack of proper control of country effects and predeterminedness.

The worry is that their estimates have finite sample downward biases in the GDP autoregressive coefficient that translate into upward biases in estimated convergence rates, as noted by Bond, Hoeffler, and Temple (2001).

We obtained the Caselli et al. data and re-estimated an augmented Solow model of the form given in (5.9) with the new projection-restricted IV estimator. We have found a smaller convergence rate of about 4 percent, but imprecisely estimated (Table 4). ${ }^{14}$ From a substantive point of view, however, explaining the uncovered heterogeneity in steady state income levels seems at least as crucial as finding good estimates of the convergence coefficient.

\footnotetext{
${ }^{13}$ See Barro and Sala-i-Martin (1995) for details and further references.

${ }^{14}$ We use similar data as Caselli et al. and Bond et al. Our sample is the same as the one in Table 2 of Bond et al., except for the exclusion of 9 countries and 17 observations in order to have a balanced panel.
} 
The standard errors reported in Table 4 were calculated from formula (7.10) with $r=T-2$. Using smaller values of $r$ made very little difference. Further exploration of the effects on the estimates and standard errors of using optimal instruments based on higher-order VAR models would be of some interest.

\section{Concluding Remarks}

We developed a new methodology for instrumental variable estimation of panel data models with general predetermined or endogenous explanatory variables. The suggested instruments are linear forecasts of the explanatory variables constructed under the assumption that the vector of conditioning variables follows a panel VAR process. These are linear combinations of all available lags as in ordinary GMM, but with the crucial difference that the number of first-stage coefficients is kept constant regardless of the value of $T$. We show analytically and through Monte Carlo simulations that this fundamentally alters the properties of the estimators in double asymptotics and finite samples. The new estimators eliminate double-asymptotic biases while retaining similar robustness and optimality properties as GMM in fixed $T$ environments.

Specific discussions on unbalanced panels and higher-order auxiliary models are clearly of practical importance, but they are left for future work. GMM is not well suited to cope with the complications derived from typical patterns of unbalancedness or rotation in firm and household panels, which magnify the number of first stage coefficients. In the GMM context this has been avoided at the expense of introducing ad-hoc restrictions in the form of the cross-sectional projections across sub-panels - see Arellano and Bond (1991) for discussion. In contrast, projection-restricted IV estimators offer the possibility of a coherent specification of optimal instruments across sub-panels based on a small number of common coefficients. 


\section{Appendix}

\section{A. Double-Asymptotic Results}

\section{Proof of Theorem 1}

We shall use the following implication of Assumption 1. For all $r, s \geq 0$ we have

$$
E_{t}\left(x_{i(t+r)} \varepsilon_{i(t+s)}\right)=E\left(x_{i(t+r)} \varepsilon_{i(t+s)}\right) .
$$

To see this note that

$$
\begin{aligned}
E\left(w_{i(t+r)} w_{i(t+s)}^{\prime} \mid w_{i}^{t}, \mu_{i}\right) & =\mu_{i} \mu_{i}^{\prime}+\sum_{j=0}^{\infty} \sum_{k=0}^{\infty} \Psi_{j} E\left(\zeta_{i(t+r-j)} \zeta_{i(t+s-k)}^{\prime} \mid \zeta_{i}^{t}, \mu_{i}\right) \Psi_{k}^{\prime} \\
& =\mu_{i} \mu_{i}^{\prime}+\sum_{j=0}^{\infty} \sum_{k=0}^{\infty} \Psi_{j} E\left(\zeta_{i(t+r-j)} \zeta_{i(t+s-k)}^{\prime}\right) \Psi_{k}^{\prime} .
\end{aligned}
$$

By the law of iterated expectations

$$
\begin{aligned}
E\left(w_{i(t+r)} w_{i(t+s)}^{\prime} \mid z_{i}^{t}\right) & =E\left[E\left(w_{i(t+r)} w_{i(t+s)}^{\prime} \mid w_{i}^{t}, \mu_{i}\right) \mid z_{i}^{t}\right] \\
& =E\left(w_{i(t+r)} w_{i(t+s)}^{\prime}\right)+E_{t}\left(\mu_{i} \mu_{i}^{\prime}\right)-E\left(\mu_{i} \mu_{i}^{\prime}\right) .
\end{aligned}
$$

Equation (A.1) holds because $\varepsilon_{i t}$ does not have an individual effect (i.e the first component of $\mu_{i}$ is zero).

Let us examine the form of $E\left(X^{* \prime} M \varepsilon^{*}\right)$. We have

$$
E\left(X^{* \prime} M \varepsilon^{*}\right)=\sum_{t=1}^{T-1} E\left(X_{t}^{* \prime} M_{t} \varepsilon_{t}^{*}\right)=m \sum_{t=1}^{T-1} t E\left(x_{i t}^{*} \varepsilon_{i t}^{*}\right) .
$$

To see this note that

$$
\begin{aligned}
E\left(X_{t}^{* \prime} M_{t} \varepsilon_{t}^{*}\right) & =\left\{E\left(x_{\ell t}^{* \prime} M_{t} \varepsilon_{t}^{*}\right)\right\}=\left\{E \operatorname{tr}\left[M_{t} E_{t}\left(\varepsilon_{t}^{*} x_{\ell t}^{* \prime}\right)\right]\right\} \\
& =m t E\left(\varepsilon_{i t}^{*} x_{i t}^{*}\right) .
\end{aligned}
$$

The last equality comes from (A.1), $\operatorname{tr}\left(M_{t}\right)=m t$, and the fact that due to crosssectional independence:

$$
E_{t}\left(\varepsilon_{t}^{*} x_{\ell t}^{* \prime}\right)=\left(\begin{array}{ccc}
E_{t}\left(\varepsilon_{1 t}^{*} x_{\ell 1 t}^{*}\right) & \ldots & E_{t}\left(\varepsilon_{1 t}^{*} x_{\ell N t}^{*}\right) \\
\vdots & \ddots & \vdots \\
E_{t}\left(\varepsilon_{N t}^{*} x_{\ell 1 t}^{*}\right) & \ldots & E_{t}\left(\varepsilon_{N t}^{*} x_{\ell N t}^{*}\right)
\end{array}\right)
$$




$$
=\left(\begin{array}{ccc}
E_{t}\left(\varepsilon_{1 t}^{*} x_{\ell 1 t}^{*}\right) & \ldots & E_{t}\left(\varepsilon_{1 t}^{*}\right) E_{t}\left(x_{\ell N t}^{*}\right) \\
\vdots & \ddots & \vdots \\
E_{t}\left(\varepsilon_{N t}^{*}\right) E_{t}\left(x_{\ell 1 t}^{*}\right) & \ldots & E_{t}\left(\varepsilon_{N t}^{*} x_{\ell N t}^{*}\right)
\end{array}\right)=E_{t}\left(\varepsilon_{i t}^{*} x_{\ell i t}^{*}\right) I_{N} .
$$

Next, consider

$$
\begin{aligned}
E\left(x_{i t}^{*} \varepsilon_{i t}^{*}\right)= & \left(\frac{T-t}{T-t+1}\right)\left\{E\left(\varepsilon_{i t} x_{i t}\right)-\frac{1}{(T-t)} E\left[\varepsilon_{i t}\left(x_{i(t+1)}+\ldots+x_{i T}\right)\right]\right. \\
& -\frac{1}{(T-t)} E\left[\left(\varepsilon_{i(t+1)}+\ldots+\varepsilon_{i T}\right) x_{i t}\right] \\
& \left.+\frac{1}{(T-t)^{2}} E\left[\left(\varepsilon_{i(t+1)}+\ldots+\varepsilon_{i T}\right)\left(x_{i(t+1)}+\ldots+x_{i T}\right)\right]\right\} .
\end{aligned}
$$

Collecting terms we obtain

$$
\begin{aligned}
E\left(x_{i t}^{*} \varepsilon_{i t}^{*}\right)= & \gamma_{0}-\frac{1}{(T-t)(T-t+1)} \\
& \times\left\{(T-t) \gamma_{-(T-t)}+\ldots+\gamma_{-1}+\gamma_{1}+\ldots+(T-t) \gamma_{(T-t)}\right\} .
\end{aligned}
$$

Thus

$$
\begin{aligned}
E\left(X^{* \prime} M \varepsilon^{*}\right) & =m \sum_{t=1}^{T-1} t E\left(x_{i t}^{*} \varepsilon_{i t}^{*}\right) \\
& =m\left(\gamma_{0} \sum_{t=1}^{T-1} t-\sum_{t=1}^{T-1} \frac{t}{(T-t)(T-t+1)} B_{(T-t)}\right)
\end{aligned}
$$

where

$$
B_{(T-t)}=(T-t) \gamma_{-(T-t)}+\ldots+\gamma_{-1}+\gamma_{1}+\ldots+(T-t) \gamma_{(T-t)} .
$$

We now use the following facts:

$$
\begin{gathered}
\sum_{t=1}^{T-1} t=\frac{1}{2}(T-1) T \\
\sum_{t=1}^{T-1} \frac{t}{(T-t)(T-t+1)}=T-\sum_{t=1}^{T} \frac{1}{t}
\end{gathered}
$$




$$
\left|B_{(T-t)}\right|<\left|B_{\infty}\right|
$$

Hence, when $x_{i t}$ is endogenous $\left(\gamma_{0} \neq 0\right)$ we have $E\left(X^{* \prime} M \varepsilon^{*}\right)=O\left(m T^{2}\right)$ or

$$
E\left(\frac{X^{* \prime} M \varepsilon^{*}}{N T}\right)=O\left(\frac{m T}{N}\right) .
$$

In contrast, when $x_{i t}$ is predetermined we have $E\left(X^{* \prime} M \varepsilon^{*}\right)=O(m T)$ or

$$
E\left(\frac{X^{* \prime} M \varepsilon^{*}}{N T}\right)=O\left(\frac{m}{N}\right) .
$$

\section{B. Auxiliary VAR with Individual Effects}

\section{B.1. Sequential linear projections of the effects}

\section{Proof of Theorem 2}

I show that for the VAR(1) model presented in the main text, the linear projection of the vector of individual effects $\mu_{i}$ on $z_{i}^{t}=\left(z_{i 0}^{\prime}, \ldots, z_{i t}^{\prime}\right)^{\prime}$ is given by

$$
\begin{gathered}
E^{*}\left(\mu_{i} \mid z_{i}^{t}\right)=\left[\Omega_{\mu}^{-1}+\Upsilon_{1}^{\prime} \Gamma_{0}^{-1} \Upsilon_{1}+(I-A)^{\prime}\left(\sum_{s=1}^{t} \Omega_{s}^{-1}\right)(I-A)\right]^{-1} \\
\times\left[\left(\Omega_{\mu}^{-1} \mu-\Upsilon_{1}^{\prime} \Gamma_{0}^{-1} \tau_{0}\right)+(I-A)^{\prime} \sum_{s=1}^{t} \Omega_{s}^{-1}\left(z_{i s}-A z_{i(s-1)}\right)+\Upsilon_{1}^{\prime} \Gamma_{0}^{-1} z_{i 0}\right] \\
=\left[I+\Lambda_{0} \Upsilon_{1}+\sum_{s=1}^{t} \Lambda_{1 s}(I-A)\right]^{-1}\left[\mu+\Lambda_{0}\left(z_{i 0}-\tau_{0}\right)+\sum_{s=1}^{t} \Lambda_{1 s}\left(z_{i s}-A z_{i(s-1)}\right)\right]
\end{gathered}
$$

for $t \geq 1$, and for $t=0$ :

$$
\begin{aligned}
E^{*}\left(\mu_{i} \mid z_{i}^{t}\right) & =\left[\Omega_{\mu}^{-1}+\Upsilon_{1}^{\prime} \Gamma_{0}^{-1} \Upsilon_{1}\right]^{-1}\left[\Omega_{\mu}^{-1} \mu+\Upsilon_{1}^{\prime} \Gamma_{0}^{-1}\left(z_{i 0}-\tau_{0}\right)\right] \\
& =\left[I+\Lambda_{0} \Upsilon_{1}\right]^{-1}\left[\mu+\Lambda_{0}\left(z_{i 0}-\tau_{0}\right)\right]
\end{aligned}
$$

where $\Lambda_{0}=\Omega_{\mu} \Upsilon_{1}^{\prime} \Gamma_{0}^{-1}$ and $\Lambda_{1 s}=\Omega_{\mu}(I-A)^{\prime} \Omega_{s}^{-1}$.

The VAR model implies that

$$
z_{i t}=\left(I-A^{t}\right) \mu_{i}+\left(v_{i t}+A v_{i(t-1)}+\ldots+A^{t-1} v_{i 1}\right)+A^{t} z_{i 0} .
$$

Substituting (4.4) in (B.3) we obtain

$$
z_{i t}=\left[I+A^{t}\left(\Upsilon_{1}-I\right)\right] \mu_{i}+A^{t} \tau_{0}+z_{i t}^{*}
$$


where

$$
z_{i t}^{*}=v_{i t}+A v_{i(t-1)}+\ldots+A^{t-1} v_{i 1}+A^{t} v_{i 0}^{\dagger} .
$$

Let $G$ denote the $(t+1) m \times m$ matrix $G=\left(I_{m}, A^{\prime}, A^{2 \prime}, \ldots, A^{t \prime}\right)^{\prime}$. Then

$$
z_{i}^{t}=F \mu_{i}+G \tau_{0}+z_{i}^{* t}
$$

where

$$
F=(\iota \otimes I)+G\left(\Upsilon_{1}-I\right),
$$

$\iota$ is a $(t+1) \times 1$ vector of ones, and $z_{i}^{* t}=\left(z_{i 0}^{* \prime}, \ldots, z_{i t}^{* \prime}\right)^{\prime}$. Therefore,

$$
\begin{gathered}
E\left(z_{i}^{t}\right)=F \mu+G \tau_{0} \\
\operatorname{Var}\left(z_{i}^{t}\right)=F \Omega_{\mu} F^{\prime}+V
\end{gathered}
$$

where $V=\operatorname{Var}\left(z_{i}^{* t}\right)$. Moreover, note that

$$
\operatorname{Cov}\left(z_{i}^{t}, \mu_{i}\right)=F \Omega_{\mu} .
$$

The linear projection is given by

$$
E^{*}\left(\mu_{i} \mid z_{i}^{t}\right)=\psi_{t}+\Pi_{t}^{\prime} z_{i}^{t}
$$

where

$$
\psi_{t}=\mu-\Pi_{t}^{\prime} E\left(z_{i}^{t}\right)
$$

and

$$
\Pi_{t}=\left[\operatorname{Var}\left(z_{i}^{t}\right)\right]^{-1} \operatorname{Cov}\left(z_{i}^{t}, \mu_{i}\right) .
$$

Letting $\Omega_{\mu}=P P^{\prime}$ and $M=F P$, and using the matrix inversion lemma

$$
\left[\operatorname{Var}\left(z_{i}^{t}\right)\right]^{-1}=\left(M M^{\prime}+V\right)^{-1}=V^{-1}-V^{-1} M\left(I+M^{\prime} V^{-1} M\right)^{-1} M^{\prime} V^{-1} .
$$

Moreover, letting $V^{-1}=B^{\prime} B$ where $B$ is a block-lower triangular matrix

$$
\begin{aligned}
\Pi_{t}^{\prime} z_{i}^{t} & =\Omega_{\mu} F^{\prime}\left[V^{-1}-V^{-1} M\left(I+M^{\prime} V^{-1} M\right)^{-1} M^{\prime} V^{-1}\right] z_{i}^{t} \\
& =P M^{\prime}\left[B^{\prime} B-B^{\prime} B M\left(I+M^{\prime} B^{\prime} B M\right)^{-1} M^{\prime} B^{\prime} B\right] z_{i}^{t} \\
& =P\left[I-\left(M^{\prime} B^{\prime}\right)(B M)\left(I+M^{\prime} B^{\prime} B M\right)^{-1}\right]\left(M^{\prime} B^{\prime}\right)\left(B z_{i}^{t}\right) \\
& =P\left[I+\left(M^{\prime} B^{\prime}\right)(B M)\right]^{-1}\left(M^{\prime} B^{\prime}\right)\left(B z_{i}^{t}\right) .
\end{aligned}
$$


Since $V=\operatorname{Var}\left(z_{i}^{* t}\right)$, the matrix $B$ has to be such that $\operatorname{Var}\left(B z_{i}^{* t}\right)=I$. Therefore, since $B$ must satisfy

$$
B z_{i}^{* t}=\left(\begin{array}{c}
\Gamma_{0}^{-1 / 2} v_{i 0}^{\dagger} \\
\Omega_{1}^{-1 / 2} v_{i 1} \\
\vdots \\
\Omega_{t}^{-1 / 2} v_{i t}
\end{array}\right)
$$

it is given by

$$
B=\left(\begin{array}{cccccc}
\Gamma_{0}^{-1 / 2} & 0 & 0 & \ldots & 0 & 0 \\
-\Omega_{1}^{-1 / 2} A & \Omega_{1}^{-1 / 2} & 0 & & 0 & 0 \\
0 & -\Omega_{2}^{-1 / 2} A & \Omega_{2}^{-1 / 2} & & 0 & 0 \\
\vdots & & & \ddots & & \vdots \\
0 & 0 & 0 & & \Omega_{t-1}^{-1 / 2} & 0 \\
0 & 0 & 0 & \ldots & -\Omega_{t}^{-1 / 2} A & \Omega_{t}^{-1 / 2}
\end{array}\right)
$$

Direct multiplication gives

$$
B M=B\left[(\iota \otimes I)+G\left(\Upsilon_{1}-I\right)\right] P=\left(\begin{array}{c}
\Gamma_{0}^{-1 / 2} \Upsilon_{1} \\
\Omega_{1}^{-1 / 2}(I-A) \\
\vdots \\
\Omega_{t}^{-1 / 2}(I-A)
\end{array}\right) P
$$

and

$$
B z_{i}^{t}=\left(\begin{array}{c}
\Gamma_{0}^{-1 / 2} z_{i 0} \\
\Omega_{1}^{-1 / 2}\left(z_{i 1}-A z_{i 0}\right) \\
\vdots \\
\Omega_{t}^{-1 / 2}\left(z_{i t}-A z_{i(t-1)}\right)
\end{array}\right)
$$

Hence

$$
\left(M^{\prime} B^{\prime}\right)(B M)=P^{\prime}\left[\Upsilon_{1}^{\prime} \Gamma_{0}^{-1} \Upsilon_{1}+(I-A)^{\prime}\left(\sum_{s=1}^{t} \Omega_{s}^{-1}\right)(I-A)\right] P
$$

and

$$
\left(M^{\prime} B^{\prime}\right)\left(B z_{i}^{t}\right)=P^{\prime}\left[\Upsilon_{1}^{\prime} \Gamma_{0}^{-1} z_{i 0}+(I-A)^{\prime} \sum_{s=1}^{t} \Omega_{s}^{-1}\left(z_{i s}-A z_{i(s-1)}\right)\right] .
$$


Inserting these terms in the expression given above

$$
\begin{aligned}
\Pi_{t}^{\prime} z_{i}^{t}= & {\left[P^{-1^{\prime}} P^{-1}+\Upsilon_{1}^{\prime} \Gamma_{0}^{-1} \Upsilon_{1}+(I-A)^{\prime}\left(\sum_{s=1}^{t} \Omega_{s}^{-1}\right)(I-A)\right]^{-1} } \\
& {\left[\Upsilon_{1}^{\prime} \Gamma_{0}^{-1} z_{i 0}+(I-A)^{\prime} \sum_{s=1}^{t} \Omega_{s}^{-1}\left(z_{i s}-A z_{i(s-1)}\right)\right] . }
\end{aligned}
$$

Finally, to obtain the vector of intercepts, note that since $E\left(z_{i 0}\right)=\tau_{0}+\Upsilon_{1} \mu$ and

$$
E \sum_{s=1}^{t} \Omega_{s}^{-1}\left(z_{i s}-A z_{i(s-1)}\right)=\left(\sum_{s=1}^{t} \Omega_{s}^{-1}\right)(I-A) \mu
$$

we have

$$
\begin{aligned}
\Pi_{t}^{\prime} E\left(z_{i}^{t}\right)= & {\left[\Omega_{\mu}^{-1}+\Upsilon_{1}^{\prime} \Gamma_{0}^{-1} \Upsilon_{1}+(I-A)^{\prime}\left(\sum_{s=1}^{t} \Omega_{s}^{-1}\right)(I-A)\right]^{-1} } \\
& {\left[\Upsilon_{1}^{\prime} \Gamma_{0}^{-1} \tau_{0}+\Upsilon_{1}^{\prime} \Gamma_{0}^{-1} \Upsilon_{1} \mu+(I-A)^{\prime}\left(\sum_{s=1}^{t} \Omega_{s}^{-1}\right)(I-A) \mu\right] }
\end{aligned}
$$

Collecting terms we obtain

$$
\begin{aligned}
\psi_{t}= & \mu-\Pi_{t}^{\prime} E\left(z_{i}^{t}\right) \\
= & {\left[\Omega_{\mu}^{-1}+\Upsilon_{1}^{\prime} \Gamma_{0}^{-1} \Upsilon_{1}+(I-A)^{\prime}\left(\sum_{s=1}^{t} \Omega_{s}^{-1}\right)(I-A)\right]^{-1} } \\
& {\left[\Omega_{\mu}^{-1} \mu-\Upsilon_{1}^{\prime} \Gamma_{0}^{-1} \tau_{0}\right] }
\end{aligned}
$$

from which the result follows.

\section{A Stationary VAR(1) Process}

The stationary case is obtained as a specialization of the initial VAR model to $\tau_{0}=0, \Upsilon_{1}=I_{m}, \Omega_{t}=\Omega$ for all $t$, and $\Gamma_{0}=\sum_{j=0}^{\infty} A^{j} \Omega A^{j \prime}$, so that $\Gamma_{0}$ satisfies $\Gamma_{0}=A \Gamma_{0} A^{\prime}+\Omega$. In such case we have

$$
\begin{gathered}
E\left(w_{i}^{t}\right)=\iota \otimes \mu \\
\operatorname{Var}\left(w_{i}^{t}\right)=\left(\iota \iota^{\prime} \otimes \Omega_{\mu}\right)+V \\
\operatorname{Cov}\left(w_{i}^{t}, \mu_{i}\right)=\iota \otimes \Omega_{\mu} .
\end{gathered}
$$

Moreover, $V$ is given by

$$
V=\left(\begin{array}{llll}
\Gamma_{0} & \Gamma_{1}^{\prime} & \ldots & \Gamma_{t}^{\prime} \\
\Gamma_{1} & \Gamma_{0} & & \Gamma_{t-1}^{\prime} \\
\vdots & & \ddots & \\
\Gamma_{t} & \Gamma_{t-1} & \ldots & \Gamma_{0}
\end{array}\right)
$$


where $\Gamma_{j}=A^{j} \Gamma_{0}$. Nevertheless, the matrix $B$ in the decomposition of the inverse of $V$ is of the same form as in the general case but with constant $\Omega$.

There are two special cases of equation (4.15) that are of some interest:

1. Uncorrelated Multivariate Error Components $(A=0)$. In this case $\Gamma_{0}=\Omega$ and $\Lambda_{1}=\Lambda_{0}$, so that

$$
E_{t}\left(z_{i t}^{*}\right)=c_{t}\left[z_{i t}-E_{t}\left(\mu_{i}\right)\right]
$$

and

$$
E_{t}\left(\mu_{i}\right)=\left[I+(1+t) \Lambda_{0}\right]^{-1}\left[\mu+\Lambda_{0} \sum_{s=0}^{t} z_{i s}\right] .
$$

2. Homogeneous $\operatorname{VAR}(1)$ process $\left(\Omega_{\mu}=0\right)$ : In this case $\delta_{i t}=\delta_{i 0}=\mu$ and $\mathcal{H}_{t}=\mathcal{H}_{0}=I$, so that $E_{t}\left(\mu_{i}\right)=\mu$ and

$$
E_{t}\left(z_{i t}^{*}\right)=c_{t}\left(I-\frac{1}{T-t} \sum_{s=1}^{t} A^{s}\right)\left(z_{i t}-\mu\right) .
$$

\section{B.2. VAR Log Likelihood Given Initial Observations}

The VAR model can be written as

$$
\left(\begin{array}{ccccc}
I & 0 & \ldots & 0 & 0 \\
-A & I & & 0 & 0 \\
\vdots & & \ddots & & \vdots \\
0 & 0 & & I & 0 \\
0 & 0 & \ldots & -A & I
\end{array}\right)\left(\begin{array}{c}
z_{i 1} \\
z_{i 2} \\
\vdots \\
z_{i(T-1)} \\
z_{i T}
\end{array}\right)=\left(\begin{array}{c}
I_{m} \\
0 \\
\vdots \\
0 \\
0
\end{array}\right) A z_{i 0}+\left(\begin{array}{c}
u_{i 1} \\
u_{i 2} \\
\vdots \\
u_{i(T-1)} \\
u_{i T}
\end{array}\right)
$$

or using a compact notation

$$
\begin{aligned}
& B z_{i}=D A z_{i 0}+u_{i} \\
& u_{i}=\left(\iota \otimes \eta_{i}\right)+v_{i}
\end{aligned}
$$

where $u_{i t}=\eta_{i}+v_{i t}, \eta_{i}=(I-A) \mu_{i}, \iota$ is a vector of ones of order $T$ and $v_{i}=$ $\left(v_{i 1}^{\prime}, \ldots, v_{i T}^{\prime}\right)^{\prime}$. Thus, under time series homoskedasticity

$$
E\left(u_{i}\right)=\iota \otimes \eta
$$


and

$$
\operatorname{Var}\left(u_{i}\right)=\left(\iota \iota^{\prime} \otimes \Omega_{\eta}\right)+\left(I_{T} \otimes \Omega\right)
$$

where $\eta=(I-A) \mu$ and $\Omega_{\eta}=(I-A) \Omega_{\mu}(I-A)^{\prime}$.

The conditional density of $z_{i}$ given $z_{i 0}$ is related to that of $u_{i}$ by

$$
f\left(z_{i} \mid z_{i 0}\right)=f\left(u_{i} \mid z_{i 0}\right) \operatorname{det}(B)
$$

but $\operatorname{det}(B)=1$ because $B$ is a triangular matrix. Moreover

$$
f\left(u_{i} \mid z_{i 0}\right)=f\left(\bar{u}_{i}, u_{i}^{*} \mid z_{i 0}\right)\left|\operatorname{det}\left(H \otimes I_{m}\right)\right|
$$

where $H=\left(\iota / T, \mathcal{A}^{\prime}\right)^{\prime}$ is a $T \times T$ transformation matrix, and $\mathcal{A}$ is the $(T-1) \times T$ forward orthogonal deviations operator, that produces $\left(H \otimes I_{m}\right) u_{i}=\left(\bar{u}_{i}^{\prime}, u_{i}^{* \prime}\right)^{\prime}$. The determinant of the transformation satisfies $\left|\operatorname{det}\left(H \otimes I_{m}\right)\right|=T^{-m / 2}$, which is an irrelevant constant.

Note that

$$
E\left[\left(H \otimes I_{m}\right) u_{i}\right]=H \iota \otimes \eta=\left(\begin{array}{l}
\eta \\
0
\end{array}\right)
$$

$$
\begin{aligned}
\operatorname{Var}\left[\left(H \otimes I_{m}\right) u_{i}\right] & =\left(H \otimes I_{m}\right)\left[\left(\iota \iota^{\prime} \otimes \Omega_{\eta}\right)+\left(I_{T} \otimes \Omega\right)\right]\left(H^{\prime} \otimes I_{m}\right) \\
& =\left(H \iota \iota^{\prime} H^{\prime} \otimes \Omega_{\eta}\right)+\left(H^{\prime} H \otimes \Omega\right) \\
& =\left(\begin{array}{cc}
\Omega_{\eta}+T^{-1} \Omega & 0 \\
0 & I_{T-1} \otimes \Omega
\end{array}\right) .
\end{aligned}
$$

In order to obtain the mean and variance matrix of $\left\{\left(H \otimes I_{m}\right) u_{i} \mid z_{i 0}\right\}$ it is convenient to introduce some additional notation. Let $\Sigma_{0}=\operatorname{Var}\left(z_{i 0}\right)=\Upsilon_{1} \Omega_{\mu} \Upsilon_{1}^{\prime}+\Gamma_{0}$ and let the linear projection of $\eta_{i}$ on $z_{i 0}$ be

$$
E^{*}\left(\eta_{i} \mid z_{i 0}\right)=\phi_{0}+\Phi_{1} z_{i 0}=\left(\phi_{0}, \Phi_{1}\right)\left(\begin{array}{c}
1 \\
z_{i 0}
\end{array}\right) \equiv \Phi f_{i 0}
$$

so that $\Phi_{1}=(I-A) \Omega_{\mu} \Upsilon_{1}^{\prime} \Sigma_{0}^{-1} \cdot{ }^{15}$ Thus under joint normality of $\eta_{i}, z_{i 0}, z_{i 1}, \ldots, z_{i T}$ :

$$
E\left[\left(H \otimes I_{m}\right) u_{i} \mid z_{i 0}\right]=\left(\begin{array}{c}
\phi_{0}+\Phi_{1} z_{i 0} \\
0
\end{array}\right)
$$

\footnotetext{
${ }^{15}$ Note that

$$
\begin{aligned}
\Phi_{1} & =\operatorname{Cov}\left(\eta_{i}, w_{i 0}\right) \Sigma_{0}^{-1}=(I-A) \operatorname{Cov}\left(\mu_{i}, w_{i 0}\right) \Sigma_{0}^{-1} \\
& =(I-A) \Omega_{\mu} \Omega_{\mu}^{-1} \operatorname{Cov}\left(\mu_{i}, w_{i 0}\right) \Sigma_{0}^{-1}=(I-A) \Omega_{\mu} \Upsilon_{1}^{\prime} \Sigma_{0}^{-1} .
\end{aligned}
$$
}


and

$$
\begin{aligned}
\operatorname{Var}\left[\left(H \otimes I_{m}\right) u_{i} \mid z_{i 0}\right] & =\operatorname{Var}\left[\left(H \otimes I_{m}\right) u_{i}\right]-\operatorname{Var}\left(\begin{array}{c}
\phi_{0}+\Phi_{1} z_{i 0} \\
0
\end{array}\right) \\
& =\left(\begin{array}{cc}
\Theta_{0} & 0 \\
0 & I_{T-1} \otimes \Omega
\end{array}\right)
\end{aligned}
$$

where $\Theta_{0}=\left(\Omega_{\eta}-\Phi_{1} \Sigma_{0} \Phi_{1}^{\prime}\right)+T^{-1} \Omega^{16}$

Then, under normality

$$
\begin{aligned}
\ln f\left(z_{i 1}, \ldots, z_{i T} \mid z_{i 0}\right)= & -\frac{(T-1)}{2} \ln \operatorname{det} \Omega-\frac{1}{2} u_{i}^{* \prime}\left(I_{T-1} \otimes \Omega^{-1}\right) u_{i}^{*} \\
& -\frac{1}{2} \ln \operatorname{det} \Theta_{0}-\frac{1}{2}\left(\bar{u}_{i}-\phi_{0}-\Phi_{1} z_{i 0}\right)^{\prime} \Theta_{0}^{-1}\left(\bar{u}_{i}-\phi_{0}-\Phi_{1} z_{i 0}\right) .
\end{aligned}
$$

\section{Estimating Scalar Autoregressive Models}

Let us consider first a scalar AR(1) model with individual effects of the form

$$
\begin{gathered}
y_{i t}=\alpha y_{i(t-1)}+(1-\alpha) \mu_{i}+v_{i t}|\alpha|<1 \\
E\left(v_{i t} \mid y_{i 0}, y_{i 1}, \ldots, y_{i(t-1)}\right)=0
\end{gathered}
$$

so that in the notation of (2.1) and (2.2) $x_{i t}$ and $z_{i t}$ are both scalar variables and $x_{i t}=z_{i t}=y_{i(t-1)}$. For convenience we assume that $y_{i 0}$ is observed. In this case

$$
\frac{1}{c_{t}} E\left(x_{i t}^{*} \mid y_{i}^{t-1}\right)=y_{i(t-1)}-\frac{1}{T-t}\left[E\left(y_{i t} \mid y_{i}^{t-1}\right)+\ldots+E\left(y_{i(T-1)} \mid y_{i}^{t-1}\right)\right] .
$$

Note that since

$$
\begin{aligned}
E\left(y_{i(t+s)} \mid y_{i}^{t-1}\right) & =\alpha^{s+1} y_{i(t-1)}+\left(1+\alpha+\ldots+\alpha^{s}\right) E\left((1-\alpha) \mu_{i} \mid y_{i}^{t-1}\right) \\
& =E\left(\mu_{i} \mid y_{i}^{t-1}\right)+\alpha^{s+1}\left[y_{i(t-1)}-E\left(\mu_{i} \mid y_{i}^{t-1}\right)\right] \quad(s=0, \ldots, T-1-t),
\end{aligned}
$$

the instrument $E\left(x_{i t}^{*} \mid y_{i}^{t-1}\right)$ is just a function of $y_{i(t-1)}$ and $E\left(\mu_{i} \mid y_{i}^{t-1}\right)$ given by

$$
E\left(x_{i t}^{*} \mid y_{i}^{t-1}\right)=c_{t}\left(1-\frac{1}{T-t} \sum_{s=1}^{T-t} \alpha^{s}\right)\left[y_{i(t-1)}-E\left(\mu_{i} \mid y_{i}^{t-1}\right)\right] .
$$

\footnotetext{
${ }^{16}$ Note that $\operatorname{Var}\left(\eta_{i} \mid w_{i 0}\right)=\left(\Omega_{\eta}-\Phi_{1} \Sigma_{0} \Phi_{1}^{\prime}\right)$.
} 
In general $E\left(\mu_{i} \mid y_{i}^{T-1}\right)$ can be a nonlinear function of $y_{i}^{T-1}$, but in the auxiliary models we shall assume it coincides with the linear projection

$$
E^{*}\left(\mu_{i} \mid y_{i}^{T-1}\right)=\psi_{T-1}+\pi_{T-1}^{\prime} y_{i}^{t-1} .
$$

In view of the results of the previous section, it turns out that the coefficients of this projection are unrestricted when the unconditional variances $E\left(v_{i t}\right)=\sigma_{t}^{2}$ are allowed to change with $t$ in an unspecified way. But even in this case note that due to the law of iterated projections

$$
E^{*}\left(\mu_{i} \mid y_{i}^{t-1}\right)=E^{*}\left[E^{*}\left(\mu_{i} \mid y_{i}^{t}\right) \mid y_{i}^{t-1}\right]
$$

all the instruments $E\left(x_{i t}^{*} \mid y_{i}^{t-1}\right)$ can be written as functions of $\alpha, \psi_{T-1}$, and $\gamma_{T-1}$. This substantially reduces the number of parameters relative to the unrestricted linear projections for $E\left(x_{i t}^{*} \mid y_{i}^{t-1}\right)$ used implicitly by standard GMM, despite being based on the same auxiliary model. Nevertheless, the number of coefficients still increases with $T$.

A Strictly Stationary Auxiliary Model As an auxiliary model we may consider a strictly stationary $\mathrm{AR}(1)$ process, in which case, using the results from Appendix B, we obtain

$$
E^{*}\left(\mu_{i} \mid y_{i}^{t-1}\right)=m_{t-1}\left(y_{i}^{t-1}, \theta\right) \equiv \frac{\mu+\phi\left[(1-\alpha) \sum_{s=1}^{t-1} u_{i s}+\left(1-\alpha^{2}\right) y_{i 0}\right]}{1+\phi\left[(t-1)(1-\alpha)^{2}+1-\alpha^{2}\right]}
$$

where $\phi=\sigma_{\mu}^{2} / \sigma^{2}, \theta=(\alpha, \phi, \mu)^{\prime}$, and $u_{i s}=y_{i s}-\alpha y_{i(s-1)}$.

Letting $\lambda=\sigma_{\eta}^{2} / \sigma^{2}=(1-\alpha)^{2} \phi$, an equivalent expression is given by

$$
\begin{aligned}
E^{*}\left(\mu_{i} \mid y_{i}^{t-1}\right)= & \frac{\mu}{1+\lambda\left(t-1+\frac{1+\alpha}{(1-\alpha)}\right)} \\
& +\frac{\lambda}{1+\lambda\left(t-1+\frac{1+\alpha}{(1-\alpha)}\right)}\left[\sum_{s=0}^{t-1} y_{i s}+\frac{\alpha}{1-\alpha}\left(y_{i 0}+y_{i(t-1)}\right)\right] .
\end{aligned}
$$

Note that as $t \rightarrow \infty E^{*}\left(\mu_{i} \mid y_{i}^{t-1}\right)$ converges to the limit of $t^{-1} \sum_{s=0}^{t-1} y_{i s}$ (which is given by $\left.\mu_{i}\right)$, but for small values of $t$ the approximation of $E^{*}\left(\mu_{i} \mid y_{i}^{t-1}\right)$ by 
$E^{*}\left(\mu_{i} \mid \sum_{s=0}^{t-1} y_{i s}\right)$ may be poor, even if $y_{i t}$ is a strictly stationary process (it would only be appropriate if $\alpha=0){ }^{17}$

Using as the auxiliary model the assumptions of strict stationarity together with the linearity of $E\left(\mu_{i} \mid y_{i}^{t-1}\right)$, the instrument $E\left(x_{i t}^{*} \mid y_{i}^{t-1}\right)$ becomes a linear function of $\sum_{s=0}^{t-1} u_{i s}$ and $y_{i 0}$ with coefficients that depend exclusively on $\alpha, \phi$ and $\mu$. Since these assumptions need not be true, the previous coefficients should be understood as pseudo true values for which we use the notation $c=(a, f, m)^{\prime}$. Thus our parameterization of the instrument is given by

$$
h_{t}\left(y_{i}^{t-1}, c\right)=c_{t}\left[1-\frac{a}{1-a}\left(\frac{1-a^{T-t}}{T-t}\right)\right]\left[y_{i(t-1)}-m_{t-1}\left(y_{i}^{t-1}, c\right)\right] .
$$

An Auxiliary Model with Unrestricted Initial Conditions An alternative, more general auxiliary model that retains a fixed number of parameters is one in which the assumption of stationarity of initial observations is removed. This adds three extra parameters to the instrument function.

The previous auxiliary model assumed that the linear projection of $y_{i 0}$ on $\mu_{i}$

$$
y_{i 0}=\tau_{0}+\tau_{1} \mu_{i}+v_{i 0}
$$

was such that $\tau_{0}=0, \tau_{1}=1$ and $\operatorname{Var}\left(v_{i 0}\right)=\gamma_{0}^{2}=\sigma^{2} /\left(1-\alpha^{2}\right)$. In the alternative model $\tau_{0}, \tau_{1}$ and $\gamma_{0}^{2}$ are free parameters. Using again the results from Appendix B we obtain

$$
E^{*}\left(\mu_{i} \mid y_{i}^{t-1}\right)=m_{t-1}^{\dagger}\left(y_{i}^{t-1}, \theta\right) \equiv \frac{\left(\mu-r_{0} \tau_{1} \tau_{0}\right)+\phi(1-\alpha) \sum_{s=1}^{t-1} u_{i s}+r_{0} \tau_{1} y_{i 0}}{1+\phi(t-1)(1-\alpha)^{2}+r_{0} \tau_{1}^{2}}
$$

where $r_{0}=\sigma_{\mu}^{2} / \gamma_{0}^{2}$ and $\theta=\left(\alpha, \phi, \mu, \tau_{0}, \tau_{1}, r_{0}\right)^{\prime}$. Note that under stationarity $r_{0}=\phi\left(1-\alpha^{2}\right)$.

For large $t$ the difference between (C.6) and (C.10) will be small regardless of the values of $\tau_{0}, \tau_{1}$ and $\gamma_{0}^{2}$, but in short panels the difference may be important if the steady state distribution of the process is not a good approximation to the

\footnotetext{
${ }^{17}$ With $T=2$, we only need to consider $t=1$, and there is just one instrument given by

$$
E\left(x_{i 1}^{*} \mid y_{i 0}\right)=2^{-1 / 2} \frac{(1-\alpha)^{2}}{(1-\alpha)+\lambda(1+\alpha)}\left(y_{i 0}-\mu\right) .
$$
}


distribution of initial observations. In such a case a better choice of instrument will be

$$
h_{t}^{\dagger}\left(y_{i}^{t-1}, c\right)=c_{t}\left[1-\frac{a}{1-a}\left(\frac{1-a^{T-t}}{T-t}\right)\right]\left[y_{i(t-1)}-m_{t-1}^{\dagger}\left(y_{i}^{t-1}, c\right)\right] .
$$

$\mathbf{A R}(p)$ Processes The previous discussion can be extended to a stable autoregressive process of order $p$ :

$$
\begin{gathered}
y_{i t}=\alpha_{1} y_{i(t-1)}+\ldots+\alpha_{p} y_{i(t-p)}+\eta_{i}+v_{i t} \\
E\left(v_{i t} \mid y_{i 0}, y_{i 1}, \ldots, y_{i(t-1)}\right)=0,
\end{gathered}
$$

so that in terms of the notation of model (2.1) and (2.2), we have $x_{i t}=\left(y_{i(t-1)}, \ldots, y_{i(t-p)}\right)^{\prime}$ and $z_{i t}=y_{i(t-1)}$. For convenience we assume that $y_{i 0}, y_{i(-1)}, \ldots, y_{i(-p+1)}$ are observed (i.e. the time series dimension of the panel is $T+p$ ), and write the model in companion form

$$
x_{i(t+1)}=\Pi x_{i t}+d_{1}\left(\eta_{i}+v_{i t}\right)
$$

where $d_{1}=(1,0, \ldots, 0)^{\prime}$ of order $p$, and $\Pi$ is the $p \times p$ matrix

$$
\Pi=\left(\begin{array}{ccc}
\alpha_{1} & \ldots & \alpha_{p} \\
I_{p-1} & \vdots & 0
\end{array}\right) .
$$

Therefore,

$$
\frac{1}{c_{t}} E\left(x_{i t}^{*} \mid y_{i}^{t-1}\right)=x_{i t}-\frac{1}{T-t}\left[E\left(x_{i(t+1)} \mid y_{i}^{t-1}\right)+\ldots+E\left(x_{i T} \mid y_{i}^{t-1}\right)\right] .
$$

Moreover, since

$$
E\left(x_{i(t+s)} \mid y_{i}^{t-1}\right)=\Pi^{s} x_{i t}+\left(I_{p}+\Pi+\ldots+\Pi^{s-1}\right) d_{1} E\left(\eta_{i} \mid y_{i}^{t-1}\right) \quad(s=1, \ldots, T-t),
$$

the vector of instruments $E\left(x_{i t}^{*} \mid y_{i}^{t-1}\right)$ is a function of $x_{i t}$ and $E\left(\eta_{i} \mid y_{i}^{t-1}\right)$ given by

$$
E\left(x_{i t}^{*} \mid y_{i}^{t-1}\right)=c_{t}\left[I-\frac{1}{T-t} \Pi\left(I-\Pi^{T-t}\right)(I-\Pi)^{-1}\right]\left[x_{i t}-\iota_{p} E\left(\mu_{i} \mid y_{i}^{t-1}\right)\right]
$$


where $\eta_{i}=\left(1-\alpha_{1}-\ldots-\alpha_{p}\right) \mu_{i}{ }^{18}$

Under the strictly stationary auxiliary model, the linear projection $E^{*}\left(\mu_{i} \mid y_{i}^{t-1}\right)$ is still of the form

$$
\begin{aligned}
E^{*}\left(\mu_{i} \mid y_{i}^{t-1}\right) & =\mu+\phi \iota^{\prime}\left(\phi \iota \iota^{\prime}+V\right)^{-1}\left(y_{i}^{t-1}-\mu \iota\right) \\
& =\frac{\mu}{1+\phi\left(\iota^{\prime} V^{-1} \iota\right)}+\frac{\phi}{1+\phi\left(\iota^{\prime} V^{-1} \iota\right)} \iota^{\prime} V^{-1} y_{i}^{t-1},
\end{aligned}
$$

but now $V=V\left(\alpha_{1}, \ldots, \alpha_{p}\right)$ corresponds to the autoregressive covariance matrix of order $p$.

\footnotetext{
${ }^{18}$ Note that

$$
(I-\Pi)^{-1} d_{1} E\left(\eta_{i} \mid y_{i}^{t-1}\right)=\iota_{p} E\left(\mu_{i} \mid y_{i}^{t-1}\right)
$$

since $\left(1-\alpha_{1}-\ldots-\alpha_{p}\right) d_{1}=(I-\Pi) \iota_{p}$.
} 


\section{References}

Alonso-Borrego, C. and M. Arellano (1999): "Symmetrically Normalized Instrumental-Variable Estimation Using Panel Data", Journal of Business 86 Economic Statistics, 17, 36-49.

Alvarez, J. and M. Arellano (2003): "The Time Series and Cross-Section Asymptotics of Dynamic Panel Data Estimators", Econometrica, 71, forthcoming.

Anderson, T.W. (1971), The Statistical Analysis of Time Series, John Wiley \& Sons, New York.

Anderson, T.W. and C. Hsiao (1982): "Formulation and Estimation of Dynamic Models Using Panel Data", Journal of Econometrics, 18, 47-82.

Arellano, M. (2003): Panel Data Econometrics, Oxford University Press, Oxford.

Arellano, M. and S.R. Bond (1991): "Some Tests of Specification for Panel Data: Monte Carlo Evidence and an Application to Employment Equations", Review of Economic Studies, 58, 277-297.

Arellano, M. and O. Bover (1995): "Another Look at the Instrumental-Variable Estimation of Error-Components Models", Journal of Econometrics, 68, 2951.

Barro, R. J. and X. Sala-i-Martin (1995), Economic Growth, McGraw Hill, New York.

Blundell, R. and R. Smith (1991): "Initial Conditions and Efficient Estimation in Dynamic Panel Data Models", Annales d'Economie et de Statistique, 20/21, 109-123.

Blundell, R. and S. Bond (1998): "Initial Conditions and Moment Restrictions in Dynamic Panel Data Models", Journal of Econometrics, 87, 115-143.

Bond, S. R., A. Hoeffler, and J. Temple (2001): "GMM Estimation of Empirical Growth Models", CEPR Discussion Paper 3048, London.

Caselli, F., G. Esquivel, and F. Lefort (1996): "Reopening the Convergence Debate: A New Look at Cross-Country Growth Empirics", Journal of Economic Growth, 1, 363-389. 
Chamberlain, G. (1987): "Asymptotic Efficiency in Estimation with Conditional Moment Restrictions", Journal of Econometrics, 34, 305-334.

Chamberlain (1992): "Comment: Sequential Moment Restrictions in Panel Data", Journal of Business \& Economic Statistics, 10, 20-26.

Hahn, J. (1997): "Efficient Estimation of Panel Data Models with Sequential Moment Restrictions", Journal of Econometrics, 79, 1-21.

Holtz-Eakin, D., W. Newey and H. Rosen (1988): "Estimating Vector Autoregressions with Panel Data, Econometrica, 56, 1371-1395.

Newey, W. K. and K. D. West (1987): "A Simple, Positive Semi-Definite, Heteroskedasticity and Autocorrelation Consistent Covariance Matrix", Econometrica, 55, 703-708.

Nickell, S. (1981): "Biases in Dynamic Models with Fixed Effects", Econometrica, 49, 1417-1426.

Zeldes, S. P. (1989): "Consumption and Liquidity Constraints: An Empirical Investigation", Journal of Political Economy, 97, 305-346. 
Table 1

Employment and Wage VAR Model Panel Data of Spanish Firms

\begin{tabular}{ccccc}
\hline & WG & GMM & PML & SIV \\
\hline \multicolumn{5}{c}{$N=738, T=8$} \\
$n_{i(t-1)}$ & 0.71 & 0.86 & 1.00 & 0.93 \\
& $(0.03)$ & $(0.06)$ & & $(0.07)$ \\
$w_{i(t-1)}$ & 0.08 & 0.12 & 0.08 & 0.14 \\
& $(0.03)$ & $(0.07)$ & & $(0.08)$
\end{tabular}

Wage equation

\begin{tabular}{ccccc}
$n_{i(t-1)}$ & 0.06 & -0.03 & 0.01 & -0.02 \\
& $(0.02)$ & $(0.08)$ & & $(0.08)$ \\
$w_{i(t-1)}$ & 0.44 & 0.29 & 0.68 & 0.32 \\
& $(0.03)$ & $(0.10)$ & & $(0.10)$ \\
\hline \multicolumn{5}{c}{$N=385, T=14$} \\
$n_{i(t-1)}$ & 0.86 & 0.83 & 0.995 & 0.90 \\
& $(0.05)$ & $(0.05)$ & & $(0.05)$ \\
$w_{i(t-1)}$ & 0.26 & 0.29 & 0.28 & 0.30 \\
& $(0.09)$ & $(0.13)$ & & $(0.15)$
\end{tabular}

Wage equation

\begin{tabular}{ccccc}
$n_{i(t-1)}$ & 0.01 & -0.09 & 0.03 & -0.15 \\
& $(0.02)$ & $(0.04)$ & & $(0.05)$ \\
$w_{i(t-1)}$ & 0.45 & 0.34 & 0.62 & 0.32 \\
& $(0.07)$ & $(0.10)$ & & $(0.12)$ \\
\hline
\end{tabular}

All data in deviations from period-specific cross-sectional means.

Estimates of $\Upsilon_{1}$ using SIV estimates of $A$ :

$$
\begin{gathered}
(N=738, T=8) \text { sample: } \\
\widehat{\Upsilon}_{1(738)}=\left(\begin{array}{cc}
0.863 & 0.155 \\
0.008 & 0.849
\end{array}\right) \\
(N=385, T=14) \text { sample: } \\
\widehat{\Upsilon}_{1(385)}=\left(\begin{array}{cc}
0.983 & 0.441 \\
-0.004 & 0.905
\end{array}\right) .
\end{gathered}
$$


Table 2

Monte Carlo Simulations for the VAR Model

\begin{tabular}{|c|c|c|c|c|c|c|c|c|}
\hline & \multicolumn{2}{|c|}{ WG } & \multicolumn{2}{|c|}{ GMM } & \multicolumn{2}{|c|}{ PML } & \multicolumn{2}{|c|}{ SIV } \\
\hline & median & mae & median & mae & median & mae & median & mae \\
\hline \multicolumn{9}{|c|}{$N=738, T=8$} \\
\hline$a_{11}$ & 0.48 & 0.32 & 0.72 & 0.08 & 0.80 & 0.02 & 0.80 & 0.05 \\
\hline$a_{12}$ & 0.09 & 0.06 & 0.13 & 0.03 & 0.15 & 0.02 & 0.15 & 0.03 \\
\hline$a_{21}$ & 0.03 & 0.03 & 0.01 & 0.04 & 0.00 & 0.01 & -0.01 & 0.05 \\
\hline$a_{22}$ & 0.12 & 0.18 & 0.29 & 0.03 & 0.30 & 0.01 & 0.30 & 0.03 \\
\hline \multicolumn{9}{|c|}{$N=385, T=14$} \\
\hline$a_{11}$ & 0.63 & 0.17 & 0.74 & 0.06 & 0.80 & 0.01 & 0.80 & 0.03 \\
\hline$a_{12}$ & 0.12 & 0.03 & 0.13 & 0.02 & 0.15 & 0.01 & 0.15 & 0.02 \\
\hline$a_{21}$ & 0.02 & 0.02 & 0.01 & 0.02 & -0.00 & 0.01 & -0.00 & 0.02 \\
\hline$a_{22}$ & 0.21 & 0.09 & 0.29 & 0.02 & 0.30 & 0.01 & 0.30 & 0.02 \\
\hline \multicolumn{9}{|c|}{$N=738, T=8$} \\
\hline \multicolumn{9}{|c|}{ Data with trend in variance } \\
\hline$a_{11}$ & 0.50 & 0.30 & 0.70 & 0.11 & 0.86 & 0.06 & 0.81 & 0.07 \\
\hline$a_{12}$ & 0.11 & 0.04 & 0.12 & 0.04 & 0.17 & 0.02 & 0.16 & 0.04 \\
\hline$a_{21}$ & 0.03 & 0.03 & 0.01 & 0.05 & -0.01 & 0.01 & -0.01 & 0.06 \\
\hline$a_{22}$ & 0.11 & 0.19 & 0.29 & 0.03 & 0.31 & 0.02 & 0.30 & 0.03 \\
\hline
\end{tabular}

1000 replications. mae is median absolute error. 
Table 3

Monte Carlo Simulations for the VAR Model

\begin{tabular}{|c|c|c|c|c|c|c|c|c|}
\hline & \multicolumn{2}{|c|}{ WG } & \multicolumn{2}{|c|}{ GMM } & \multicolumn{2}{|c|}{ PML } & \multicolumn{2}{|c|}{ SIV } \\
\hline & median & mae & median & mae & median & mae & median & mae \\
\hline \multicolumn{9}{|c|}{$N=200, T=8$} \\
\hline$a_{11}$ & 0.48 & 0.32 & 0.59 & 0.21 & 0.80 & 0.05 & 0.80 & 0.09 \\
\hline$a_{12}$ & 0.09 & 0.06 & 0.08 & 0.07 & 0.15 & 0.03 & 0.16 & 0.06 \\
\hline$a_{21}$ & 0.03 & 0.03 & 0.03 & 0.07 & -0.00 & 0.02 & 0.00 & 0.09 \\
\hline$a_{22}$ & 0.12 & 0.18 & 0.27 & 0.05 & 0.30 & 0.02 & 0.30 & 0.05 \\
\hline \multicolumn{9}{|c|}{$N=100, T=6$} \\
\hline$a_{11}$ & 0.35 & 0.45 & 0.40 & 0.40 & 0.80 & 0.10 & 0.80 & 0.22 \\
\hline$a_{12}$ & 0.08 & 0.07 & 0.04 & 0.13 & 0.15 & 0.06 & 0.15 & 0.12 \\
\hline$a_{21}$ & 0.04 & 0.05 & 0.04 & 0.14 & -0.00 & 0.05 & -0.01 & 0.19 \\
\hline$a_{22}$ & 0.04 & 0.26 & 0.22 & 0.10 & 0.30 & 0.05 & 0.29 & 0.11 \\
\hline \multicolumn{9}{|c|}{$N=50, T=15$} \\
\hline$a_{11}$ & 0.64 & 0.16 & 0.62 & 0.18 & 0.80 & 0.04 & 0.80 & 0.06 \\
\hline$a_{12}$ & 0.12 & 0.04 & 0.10 & 0.06 & 0.15 & 0.04 & 0.15 & 0.05 \\
\hline$a_{21}$ & 0.02 & 0.03 & 0.02 & 0.04 & 0.00 & 0.02 & 0.00 & 0.06 \\
\hline$a_{22}$ & 0.22 & 0.09 & 0.24 & 0.06 & 0.30 & 0.03 & 0.30 & 0.04 \\
\hline
\end{tabular}


Table 4

Augmented Solow Model

$$
N=92, T=5
$$

\begin{tabular}{ccccc}
\hline & OLS & WG & GMM & SIV \\
\hline $\begin{array}{c}1+\beta) \\
(\text { s.e. })\end{array}$ & 0.947 & 0.680 & 0.698 & 0.808 \\
& $(0.017)$ & $(0.057)$ & $(0.107)$ & $(0.248)$ \\
$\ln \left(e n r_{t}\right)$ & 0.035 & -0.049 & -0.140 & -0.114 \\
$($ s.e. $)$ & $(0.014)$ & $(0.029)$ & $(0.066)$ & $(0.229)$ \\
& & & & \\
$\ln \left(s_{t}\right)$ & 0.081 & 0.138 & 0.144 & 0.090 \\
$($ s.e. $)$ & $(0.017)$ & $(0.039)$ & $(0.055)$ & $(0.075)$ \\
$\ln \left(n_{t}+g+d\right)$ & -0.094 & -0.033 & 0.230 & 0.227 \\
$($ s.e. $)$ & $(0.053)$ & $(0.152)$ & $(0.339)$ & $(1.384)$ \\
& & & & \\
$\operatorname{Implied~} \lambda$ & 0.011 & 0.077 & 0.072 & 0.043 \\
$($ s.e. $)$ & $(0.004)$ & $(0.017)$ & $(0.031)$ & $(0.062)$ \\
\hline
\end{tabular}

All data in deviations from period-specific cross-sectional means. Data for 5-year intervals 1960-1985.

s.e. robust to heterosk. \& autocorrelation, $m=T-2$.

$n=$ population growth rate; $g=$ rate of technical change;

$d=$ rate of depreciation of physical capital $(g+d=0.05)$;

$s=$ saving rate; enr $=$ secondary-school enrollment rate. 


\section{CEMFI WORKING PAPERS}

0001 Enrique Sentana: "Factor representing portfolios in large asset markets".

0002 Ana Fernandes: "Altruism with endogenous labor supply".

0003 Maria Gutiérrez-Urtiaga: "Managers and directors: A model of strategic information transmission".

0004 Gilles Saint-Paul and Samuel Bentolila: "Will EMU increase Eurosclerosis?".

0005 Giorgio Calzolari, Gabriele Fiorentini and Enrique Sentana: "Constrained EMM and indirect inference estimation".

0006 José M. Campa, P. H. Kevin Chang and James F. Refalo: "An options-based analysis of emerging market exchange rate expectations: Brazil's Real plan, 1994-1999".

0007 Gabriele Fiorentini, Enrique Sentana and Giorgio Calzolari: "The score of conditionally heteroskedastic dynamic regression models with Student $t$ innovations, and an LM test for multivariate normality".

0008 Pedro Albarran: "Income uncertainty and precautionary saving: Evidence from household rotating panel data".

0009 Claudio Michelacci and Javier Suarez: "Business creation and the stock market".

0010 Samuel Bentolila and Andrea Ichino: "Unemployment and consumption: Are job losses less painful near the Mediterranean?".

0011 Juan Ayuso and Rafael Repullo: "A model of the open market operations of the European Central Bank".

0012 Gerard Llobet, Hugo Hopenhayn and Matthew Mitchell: "Rewarding sequential innovators: Prizes, patents and buyouts".

0013 María Gutiérrez: "A contractual approach to the regulation corporate directors' fiduciary duties".

0014 María Gutiérrez: "An economic analysis of corporate directors' fiduciary duties".

0015 Rafael Repullo: "A model of takeovers of foreign banks".

0016 Manuel Arellano and Bo Honoré: "Panel data models: Some recent developments".

0101 Manuel Arellano: "Discrete choices with panel data".

0102 Gerard Llobet: "Patent litigation when innovation is cumulative".

0103 Andres Almazán and Javier Suarez: "Managerial compensation and the market reaction to bank loans".

0104 Juan Ayuso and Rafael Repullo: "Why did the banks overbid? An empirical model of the fixed rate tenders of the European Central Bank".

0105 Enrique Sentana: "Mean-Variance portfolio allocation with a Value at Risk constraint".

0106 José Antonio García Martín: "Spot market competition with stranded costs in the Spanish electricity industry".

0107 José Antonio García Martín: "Cournot competition with stranded costs".

0108 José Antonio García Martín: "Stranded costs: An overview”. 
0109 Enrico C. Perotti and Javier Suárez: "Last bank standing: What do I gain if you fail?".

0110 Manuel Arellano: "Sargan's instrumental variable estimation and GMM".

0201 Claudio Michelacci: "Low returns in R\&D due to the lack of entrepreneurial skills".

0202 Jesús Carro and Pedro Mira: "A dynamic model of contraceptive choice of Spanish couples".

0203 Claudio Michelacci and Javier Suarez: "Incomplete wage posting".

0204 Gabriele Fiorentini, Enrique Sentana and Neil Shephard: "Likelihood-based estimation of latent generalised ARCH structures".

0205 Guillermo Caruana and Marco Celentani: "Career concerns and contingent compensation".

0206 Guillermo Caruana and Liran Einav: "A theory of endogenous commitment".

0207 Antonia Díaz, Josep Pijoan-Mas and José-Víctor Ríos-Rull: "Precautionary savings and wealth distribution under habit formation preferences".

0208 Rafael Repullo: "Capital requirements, market power and risk-taking in banking".

0301 Rubén Hernández-Murillo and Gerard Llobet: "Patent licensing revisited: Heterogeneous firms and product differentiation".

0302 Cristina Barceló: "Housing tenure and labour mobility: A comparison across European countries".

0303 Víctor López Pérez: "Wage indexation and inflation persistence".

0304 Jesús M. Carro: "Estimating dynamic panel data discrete choice models with fixed effects".

0305 Josep Pijoan-Mas: "Pricing risk in economies with heterogenous agents and incomplete markets".

0306 Gabriele Fiorentini, Enrique Sentana and Giorgio Calzolari: "On the validity of the Jarque-Bera normality test in conditionally heteroskedastic dynamic regression models".

0307 Samuel Bentolila and Juan F. Jimeno: "Spanish unemployment: The end of the wild ride?".

0308 Rafael Repullo and Javier Suarez: "Loan pricing under Basel capital requirements".

0309 Matt Klaeffling and Victor Lopez Perez: "Inflation targets and the liquidity trap".

0310 Manuel Arellano: "Modelling optimal instrumental variables for dynamic panel data models". 